\title{
Kulturtheorie, Systemtheorie und das sozialtheoretische Muster der Innen-Außen-Differenz
}

\section{Andreas Reckwitz}

Universität Hamburg, Institut für Soziologie, Allendeplatz 1, 20146 Hamburg

Zusammenfassung: Niklas Luhmanns konstruktivistische Systemtheorie und die neueren Ansätze einer kulturtheoretischen Analyse wissensangeleiteter sozialer Praktiken, wie sie exemplarisch bei Pierre Bourdieu und Anthony Giddens präsentiert wird, vollziehen die 'interpretative Wende' in den Sozialwissenschaften in einer jeweils konträren Theoriearchitektur. Luhmann baut mit seiner Leitunterscheidung zwischen psychischen Systemen und sozialen Systemen in Anlehnung an Descartes, Husserl und Durkheim auf einer Innen-Außen-Differenz zwischen Bewußtsein und Sozialwelt auf. Die Kulturtheorien bei Bourdieu und Giddens distanzieren sich hingegen in Anlehnung an Saussure und den späten Wittgenstein von dieser Innen-Außen-Differenz und gehen stattdessen von der analytischen Leitdifferenz zwischen Wissensstrukturen und Handlungspraxis aus. Rekonstruiert man Systemtheorie und Kulturtheorien in dieser Weise, verschieben sich die Fronten gängiger Theoriekritik: Die Kulturtheorien erscheinen nicht als 'individualistisch', sondern umgekehrt als Vertreter eines sozialen Regelholismus. Demgegenüber besitzt Luhmanns vorgeblicher Holismus im Begriff des psychischen Systems eine individualistische Kehrseite und sieht sich mit der kulturtheoretischen Kritik konfrontiert, soziales 'Wissen' auf Semantik zu reduzieren.

Um das Feld vergangener und gegenwärtiger Sozialtheorien zu systematisieren, schlug George Ritzer Mitte der 1970er Jahre ein triadisches Schema vor, das die drei möglichen 'Metatheorien' der Sozialwissenschaft voneinander unterscheiden soll: das sich von Durkheims Konzeption einer Sozialphysik herleitende 'social facts'-Paradigma, das von der behavioristischen Psychologie beeinflußte 'social behavior'-Paradigma, schließlich das klassisch insbesondere in Max Webers Konzept einer Soziologie der 'Sinnzusammenhänge' verfochtene kulturwissenschaftliche 'social definition'-Paradigma. Ritzer zufolge existierten in der Geschichte der Sozialwissenschaften alle drei Denkschulen durchgängig nebeneinander, allerdings begleitet von gelegentlichen Gewichtsverschiebungen. (Ritzer 1975)

Kaum überraschend haben, seitdem Ritzer seine Theoriesystematik formulierte, die theoretischen Gewichte sich tatsächlich verschoben und zudem die einzelnen Theorierichtungen ihre Gestalt geändert: Das 'social facts'-Paradigma verlor sowohl in der Version einer naturalistischen Soziologie sozialer Regelmäßigkeiten als auch in der Form eines am 'Homo sociologicus' orientierten Strukturfunktionalismus an Boden. Das methodologischindividualistische 'social behavior'-Lager hat durch die Ausbreitung des Rational Choice Ansatzes in Soziologie und Politikwissenschaft eine ungeahnte Stärkung erfahren und sich damit von der Verhaltens- zur Handlungstheorie gewandelt. Die tiefgreifendste Gewichtsverschiebung ist aber wohl in der Expansion derjenigen Sozialtheorien,
Wissenschaftstheorien der Sozialwissenschaft und Methodologien zu sehen, die sich im weitesten Sinne unter dem Dach von Ritzers 'social definition'Paradigmas wiederfinden, allerdings mit diesem Begriff wohl kaum mehr zu erfassen sind. Vielmehr kann man eine weitreichende 'interpretative Wende' in den neueren Sozialwissenschaften ( $\mathrm{Ra}$ binow/ Sullivan 1979; Bohman et al. 1991), eine Entwicklung zu 'kulturtheoretischen' oder 'konstruktivistischen' Denkansätzen (Chaney 1994; Schmidt 1987a) diagnostizieren, die weit über den mikrosoziologischen 'interpretative approach' (T. Wilson) der 1960er Jahre hinausreicht.

Die verschiedenen Spielarten einer solchen 'sinnorientierten' Sozialwissenschaft eint eine gemeinsame Grundposition: die Annahme einer sinnhaften Konstitution der sozialen Welt. Grundlegend für die Arbeit der Sozialwissenschaften muß aus dieser Perspektive die Einsicht sein, daß anders als die Welt der Natur die Reproduktion der Sozialwelt auf der Existenz von kollektiven, handlungs- und kommunikationsanleitenden Bedeutungsmustern beruht, die bereits ganz ohne Zutun des Wissenschaftlers wirken. Diese Sinnmuster und die Interpretationsakte, die auf ihrer Grundlage vollzogen werden, lassen sich damit nicht als bloße - gar die eigentlichen 'sozialen Tatsachen' verzerrenden - Epiphänomene abtun, sondern bilden die Basis, auf der die soziale Welt möglich ist und auf der allein sie verstehbar wird. Damit stellt sich im übrigen auch die Wissenschaft, als Teil der Sozialwelt begriffen, zwangsläufig als eine interpretative, auf spezifischen Sinnschemata aufbauende Tätigkeit dar. 
Der in der gegenwärtigen deutschsprachigen Diskussion prominenteste Ansatz, der in radikaler Weise auf dem Gedanken einer sinnhaften Konstitution der sozialen Welt aufbaut, ist wohl Niklas Luhmanns systemtheoretischer Konstruktivismus, seine Theorie selbstreferentiell beobachtender, sich autopoietisch reproduzierender Sinnsysteme. Alternativ dazu und ohne gegenseitige Bezugnahme haben sich insbesondere in der angelsächsischen und französischen Theoriediskussion eine Reihe von Sozialtheorien ausgebildet, die vor allem in Anlehnung an Wittgenstein und an den Strukturalismus soziale Praktiken als angeleitet von kollektiven Wissensstrukturen und deren Interpretationsleistungen betrachten. Diese Theorien wissensabhängiger sozialer Praktiken, die in den Ansätzen von Pierre Bourdieu und Anthony Giddens wohl ihre elaborierteste Form annehmen, sollen unter der Überschrift 'Kulturtheorien' zusammengefaßt werden.

Je nachdem, aus welcher Perspektive man diese beiden Theorieoptionen betrachtet, scheinen sie sehr viel oder sehr wenig gemeinsam zu haben. Zunächst gilt es festzuhalten, daß sowohl die Theorie selbstreferentieller Beobachtungssysteme als auch die Theorien wissensangeleiteter sozialer Praktiken 'Sinn' als Grundbegriff der Sozialwissenschaften voraussetzen und damit die Sozialität menschlicher Welt in sozialen Konstruktions- und Interpretationsleistungen begründet sehen. Bei Luhmann sind die sozialen und psychischen Systeme Sinnsysteme: ihre Kommunikationen und Gedanken operieren auf der Grundlage von spezifischen Beobachtungsschemata. Für die Kulturtheorien werden soziale Praktiken durch die kollektiven Sinnschemata der Akteure, über die diese in Form eines impliziten Wissens verfügen, begrenzt und ermöglicht. Wenn Luhmann die Aufgabe der Sozialwissenschaften primär als die einer Beobachtung von Beobachtungen definiert, so ist im Prinzip Ähnliches gemeint wie bei Giddens, wenn dieser von einer Konstellation 'doppelter Hermeneutik' spricht (Luhmann 1990: 68ff; Giddens 1976: 163ff): In beiden Fällen werden die Sozialwissenschaften auf eine Rekonstruktion von sozialen Sinnzusammenhängen festgelegt - dies ist die fundamentale Gemeinsamkeit zwischen der konstruktivistischen Systemtheorie und den kulturtheoretischen Ansätzen.

Andererseits sind nun aber die Differenzen in der Theoriearchitektur dieser beiden in den weiteren Kontext der 'interpretativen Wende' einzuordnenden Ansätze beträchtlich. Es wäre allerdings irreführend, diese Differenzen in der gängigen Unter- scheidung zwischen 'individualistischen' Handlungstheorien und 'holistischen' Systemtheorien begründet zu sehen. Die Unterschiede zwischen der Theoriearchitektur der Kulturtheorien und der konstruktivistischen Systemtheorie sind vielmehr primär in der konträren Behandlung von Innen-Außen-Differenzen zu suchen.

Kulturtheorien und konstruktivistische Systemtheorie gehen von unterschiedlichen begrifflichen Leitdifferenzen aus. Die Leitdifferenz der Kulturtheorien ist die zwischen Wissensstruktur und Handlungspraxis, wobei letztere in erstere eingebettet erscheint. Die Leitdifferenz der Systemtheorie ist hingegen die zwischen sozialen Systemen und psychischen Systemen, die beide als autopoietische, sich selbst produzierende Operationssequenzen gedacht sind. Vor dem Hintergrund dieser Begriffsentscheidungen stehen Kulturtheorie und Systemtheorie in zwei unterschiedlichen, ja letztlich einander entgegengesetzten Relationen zu jener philosophisch und sozialtheoretisch einflußreichen Tradition des Denkens in einer InnenAußen-Differenz zwischen 'Bewußtsein' und 'AuBenwelt', die von Descartes' Subjekt-Objekt-Dualismus zu Husserls Differenz zwischen ego cogito und cogitatum einerseits, zu Durkheims Dualismus zwischen Individuum und sozialen Tatsachen andererseits reicht.

Die Kulturtheorien unterschiedlichster Provenienz, am deutlichsten in den Ansätzen von Pierre Bourdieu und Anthony Giddens, situieren sich mit ihrem Konzept wissensangeleiteter sozialer Praktiken jenseits des Dualismus zwischen Innenwelt der Bewußtsein und (sozialer) Außenwelt (1). Luhmanns Systemtheorie hingegen knüpft in ihrer Unterscheidung zwischen individuellen psychischen Systemen und sozialen Systemen in flexibilisierter Form ausdrücklich an diese Innen-AußenDifferenz an (2). Rekonstruiert man die Theorien in dieser Weise, lassen sich gängige gegenseitige Kritikpunkte nicht aufrechterhalten. Die Kulturtheorien lassen sich nicht als methodologisch-individualistisch einordnen, sondern im Gegenteil als Formen eines sozialen Regelholismus. Umgekehrt besitzt Luhmanns 'holistische' Theorie sozialer Systeme eine 'individualistische' Kehrseite in ihrem

\footnotetext{
I Ähnliches, aber nicht Identisches ist gemeint, wenn Plessner von einer Tradition 'cartesianischer Sozialwissenschaft' spricht: Hier wird die sozialwissenschaftlich einflußreiche cartesianische Innen-Außen-Differenz primär als eine solche zwischen menschlichem BewuBtsein und menschlichem Körper interpretiert. (Plessner 1928: Kap. 2). Vgl. dazu auch Grathoff 1989: Kap. 2.
} 
Konzept des psychischen Systems. Aus der Sicht der Kulturtheorien muß sie sich dann der Frage stellen, ob die Innen-Außen-Differenz zwischen psychischen und sozialen Systemen nicht einer Eingrenzung des - kulturtheoretisch zum Verständnis sozialer Reproduktion zentralen - Konzepts des sozialen Wissens auf 'Semantik' Vorschub leistet (3).

\section{Die Kulturtheorien jenseits der Innen- Außen-Differenz}

Der Begriff 'Kulturtheorie' wird in der sozialwissenschaftlichen Theoriediskussion nicht in eindeutiger Weise verwendet. 'Kultur' ist ein chronisch vieldeutiger Begriff geblieben, ${ }^{2}$ und eine 'Kulturtheorie' im Singular gibt es nicht. Man sollte vielmehr vorsichtiger von einer kulturtheoretischen Perspektive sprechen, die man idealtypisch herausfiltern kann. Eine solche hat sich in der zweiten Hälfte, insbesondere im letzten Viertel des 20. Jahrhunderts in den Arbeiten von Autoren wie Pierre Bourdieu, Michel Foucault, Ulrich Oevermann, Mary Douglas, Alfred Schütz, Clifford Geertz, Erving Goffman, Anthony Giddens oder Charles Taylor in mannigfachen Variationen herausgeschält. Der Kerngedanke der kulturtheoreti-

${ }^{2}$ Der Begriff Kultur wird in der sozialtheoretisch relevanten Begriffsgeschichte vor allem in vier verschiedenen Bedeutungen verwendet: 1 ) der normative Kulturbegriff, der sich in der Aufklärung ausbildet (Pufendorf, Adelung. Kant) und bis in die Kulturkritik des 19. und 20. Jahrhunderts reicht (M. Arnold, G. Simmel, A. Weber): hier erscheint 'Kultur' als eine zivilisatorisch oder moralisch ausgezeichnete menschliche Lebensform; 2) der holistische Kulturbegriff, der von Herder herkommend sich in der Kulturanthropologie und den cultural studies fortsetzt: Kultur ist die historisch-spezifische gesamte Lebensform eines Kollektivs in Differenz zu anderen Kollektiven; 3) der differenzierungstheoretische Kulturbegriff: Kultur ist hier ein gesellschaftlich ausdifferenziertes Teilsystem, in dem in institutionalisierter Form Kunst, Bildung und Wissenschaft situiert sind (vgl. Parsons' pattern maintenancebzw. Treuhandsystem); 4) der sinn- und wissensorientierte Kulturbegriff: in der neueren sozialtheoretischen Diskussion wird Kultur als eine analytische Dimension identifiziert, die sozialen Praktiken und ihren Produkten zugrundeliegt bzw. dadurch erzeugt wird, als die Dimension der Bedeutungen, wie sie in den praxisanleitenden Wissensbeständen auf Dauer gestellt werden: ,... a new view of culture as shared knowledge - not as people's customs and artifacts and oral traditions, but what they must know in order to act as they do, making things they make, and interpret their experiences in the distinctive way they do." (Quinn/ Holland 1987: 4) schen Perspektive ist ebenso einfach wie folgenreich: Die alltäglichen sozialen Praktiken $^{3}$ des Menschen - kommunikativer und nicht-kommunikativer Art - werden ermöglicht und eingeschränkt durch kollektive Sinnmuster, durch meist implizit bleibende Wissensstrukturen, ${ }^{4}$ die in der sozialen Praxis eingesetzt werden und diese anleiten.

In die neuere kulturtheoretische Diskussion gehen strukturalistische und poststrukturalistische, sprachspieltheoretische, pragmatistische und hermeneutisch-interpretative Theorieelemente in verschiedenartigen Spielarten ein. In exemplarischer Weise ist der kulturtheoretische Argumentationskern, in dem die strukturalistische und die interpretativ-verstehende Theorietradition letztlich konvergieren, jedoch wohl in Pierre Bourdieus 'Theorie der Praxis' und in Anthony Giddens' 'Theorie der Strukturierung' skizziert worden, die trotz gewisser Differenzen ein gemeinsames kulturtheoretisches Vokabular bieten. Grundlegend ist hier die Annahme, daß menschliches Handeln nicht als ein Konglomerat diskreter, intentionaler Einzelhandlungen, sondern als kontinuierliche Sequenz von Praktiken begriffen - nur vor dem Hintergrund kollektiver Wissensbestände zu verstehen ist, welche wiederum keine praxisenthobenen Ideenwelten, sondern ein know how von Deutungsregeln der Akteure darstellen. Diese Wissensstrukturen geben den Handelnden kaum je verbalisierte Sinnmuster an die Hand, die ihnen dazu verhelfen, die Welt, ihre Handlungsumwelt, das eigene Ich, das Verhalten anderer Akteure und deren Objektivationen zu deuten. Die Sinnzuschreibungen auf der Basis der impliziten Wissensstrukturen befähigen zum Handeln und schließen gleichzeitig andere Handlungsweisen als 'undenkbar' aus. Die Relation zwischen Wissensformationen und Handeln läßt sich damit als Verhältnis von Struktur und Praxis verstehen, von generativen

\footnotetext{
${ }^{3}$ Der Begriff der 'sozialen Praktiken' hat sich in der neueren kulturtheoretischen Diskussion zur Bezeichnung von durch implizite, kollektive Wissensstrukturen ermöglichten Handlungsweisen eingebürgert, die von den Akteuren - für ihre Umwelt sichtbar - 'kompetent' hervorgebracht werden (vgl. auch Cohen 1996, kritisch dazu Turner 1994). Der Begriff wird in dieser Weise nicht nur bei Giddens und Bourdieu, sondern auch bei Foucault, den Ethnomethodologen und in den cultural studies verwendet und sollte nicht mit der Begriffsverwendung in der Tradition der normativ-kritischen sog. 'Praxisphilosophie' aus der Budapester Schule verwechselt werden.

${ }^{4}$ Zum Begriff des impliziten Wissens vgl. die Skizze von Michael Polanyi (1966: Kap. 1).
} 
Deutungsregeln und kontextspezifischer Handlungssequenz.

Zwei philosophische 'Revolutionen' des 20. Jahrhunderts sind für die sozialwissenschaftlichen Kulturtheorien im allgemeinen, für die Ansätze von Giddens und Bourdieu im besonderen wegweisend: der Strukturalismus, programmatisch von Saussure formuliert, einerseits, Wittgensteins Philosophie der 'Sprachspiele als Lebensformen', wie dieser sie in seinem Spätwerk skizziert, andererseits. Saussure stellt für den Fall der Sprache und im übertragenen Sinne für sämtliche Bedeutungssysteme fest, daß diese keine singulären Produkte einzelner Subjekte darstellen, sondern Systeme von Zeichen, die unabhängig vom Einzelnen immanent nach MaBgabe interner Form- und Bedeutungsdifferenzen strukturiert sind. Saussures Strukturalismus setzt die Präexistenz zeitlich dauerhaft bestehender Regelsysteme (langue) voraus, die sich unabhängig vom Subjekt als diesem vorangehende 'soziale Entitäten' darstellen. Gleichzeitig können die Regelsysteme jedoch nur wirksam werden, indem die (Sprech-) Handelnden über sie verfügen und damit in der Lage sind, regelentsprechende Handlungssequenzen (parole) hervorzubringen, ohne $\mathrm{da} B$ sie die von ihnen angewandten Regeln unbedingt explizit formulieren können. Der Strukturalismus geht mithin von einem generativen Status der Regelsysteme aus, die auf diese Weise 'durch die Subjekte hindurch' wirken. (Saussure 1916)

Wittgensteins Ausgangspunkt sind die 'gemeinsamen menschlichen Handlungsweisen'. Diese Lebensformen beruhen auf gemeinsamen 'Regeln' und 'Bedeutungen', die den Handelnden ein fragloses praktisches Wissen an die Hand geben, wie im entsprechenden Kontext angemessen und kompetent zu handeln ist. 'Wissen' ist zwangsläufig auf das regelmäßige Tun, auf die Praxis bezogen und wäre als praxisenthobene 'Bewußtseinsstruktur' mißverstanden. 'Wissen' und 'Bedeutungen' hängen zusammen: Das Wissen ermöglicht es den Akteuren, sich in einer für sie bedeutungsvollen, sinnhaften Welt zurechtzufinden, durchdringt selbst ihre scheinbar subjektiven Empfindungen, ohne $\mathrm{da} B$ dabei jedoch explizite Interpretationsakte vollzogen werden müßten; das sozialisierte Wissen bleibt in der Regel implizit. Der Gedanke, es könne private Regeln und Bedeutungen geben, wird von Wittgenstein zurückgewiesen: Wenn die Validität des Wissens nicht in der Welt selber fundiert sein kann, so muß sie in der Pragmatik des gemeinsamen Handelns begründet sein, nicht in individuellen Vorstellungen. (Wittgenstein 1953, 1969)
Die sozialwissenschaftliche Kulturtheorie, die in den Arbeiten von Pierre Bourdieu und Anthony Giddens präsentiert wird, schließt nun sowohl an den Strukturalismus als auch an Wittgenstein - in jeweils unterschiedlicher Gewichtung - an. ${ }^{5}$ Bourdieu geht von der strukturalistischen Differenz von langue und parole aus, die bei ihm jedoch im Sinne einer Theorie der Praxis und unter Berufung auf Wittgenstein umakzentuiert wird. Wenn in Saussures Strukturalismus die parole, die Sequenz dessen, was gesprochen wird, durch das generative Regelsystem einer langue 'erklärt' wird, dann lassen sich in Bourdieus Kulturtheorie soziale Praktiken generell - von motorischem Handeln des Leibes bis hin zu diskursiven Sprechhandeln - erklären, wenn sie als generiert durch die Habitusformationen der jeweiligen sozialen Gruppe/ Klasse erscheinen. Der Habitus ist das „System der organischen oder mentalen Dispositionen und der unbewußten Denk-, Wahrnehmungs- und Handlungsschemata, das die Erzeugung (von) Gedanken, Wahrnehmungen und Handlungen (bedingt)" (Bourdieu 1970: 40). Habitusformationen haben nach Bourdieu sowohl einen dispositionalen als auch einen dekodierenden Charakter: Sie wirken als kollektive Dispositionen zu bestimmten Handlungsformen, sie liefern nach Art eines 'Ethos' Modelle des Wünschenswerten und leiten dergestalt - scheinbar - 'subjektive' Interessen an, sie bieten als 'Sinn für...' ein kaum verbalisierbares praktisches know how dafür, wie in einzelnen Handlungsfeldern kontextadäquat $\mathrm{zu}$ handeln ist. Dekodierend wirken die Habitusformationen gleichzeitig, indem sie den Akteuren ermöglichen und erzwingen, den Objekten in ihrer Handlungsumwelt im Rahmen eines habitusspezifischen Differenzensystems spezifische Bedeutungen zuzuschreiben. (Bourdieu 1972, 1980)

Was für Bourdieu der Habitus ist, sind für Anthony Giddens im Rahmen seiner Strukturierungstheorie die Regeln des practical consciousness. Giddens schließt in erster Linie an Wittgensteins Begriffe der Regeln und des Wissens an, erst in

\footnotetext{
${ }^{5}$ Die Sozialtheorien von Bourdieu und Giddens gehen selbstverständlich nicht vollständig in der hier skizzierten kulturtheoretischen Argumentation auf, sondern reichen jeweils sehr viel weiter: Bourdieu präsentiert eine Theorie des Zusammenhangs zwischen Kapital, Habitus und Feld, Giddens eine Theorie des Zusammenhangs von Regeln, Ressourcen und unintendierten Handlungsfolgen. Gleichwohl läßt sich das kulturtheoretische Argument der Abhängigkeit sozialer Praktiken von Habitusformationen bzw. von Regeln des praktischen BewuBtseins als Kern der beiden Ansätze festmachen.
} 
zweiter Linie an den Strukturalismus (und Garfinkels Ethnomethodologie), so daß sich gegenüber Bourdieu eine stärkere Betonung der handlungskontrollierenden Kompetenz des einzelnen Akteurs gegenüber den inkorporierten Regeln ergibt. Prinzipiell ist das hier angenommene Modell von sozialen Praktiken und implizitem Regelwissen aber ähnlich: Handeln in der zeitlichen Sequenz ist unweigerlich ermöglicht und eingeschränkt durch Strukturen, durch Regeln (und Ressourcen), die gleichzeitig im Handlungsvollzug reproduziert (oder gegebenenfalls verändert) werden. Diese Regeln haben nicht den Charakter von externen, sanktionierten Normen, sondern eines vorbewußt bleibenden, impliziten Regelwissens, das in Form von 'Erinnerungsspuren' des praktischen Bewußtseins (abzugrenzen vom diskursiven Bewußtsein und vom Unbewußten) wirkt. Das Regelwissen, das die Akteure routinisiert einsetzen, liefert sowohl ein methodisches know how, das ihnen angibt, 'how to go on' als auch ein System interpretativer Schemata. Die Wissensstrukturen lassen sich für den Sozialwissenschaftler je nach Fragestellung entweder als Eigenschaft eines Bewußtseins oder Akteurs (so $\mathrm{da}$ von der Strukturdimension abstrahiert wird) oder als überindividuelle Regelstruktur (was mit einer Einklammerung der Akteure verbunden ist) begreifen. Letztendlich existieren die 'abwesenden' Wissensstrukturen jedoch allein im realen Handlungsprozeß, und umgekehrt setzt die Handlungsfähigkeit der Akteure wiederum die Verfügung über implizite Wissensstrukturen voraus. (Giddens 1979, 1984)

Mit der Differenz zwischen 'Struktur' und 'Praxis' werden keine eigenständig existierenden Sphären voneinander geschieden, sie muß bei Giddens und Bourdieu vielmehr als eine analytisch-heuristische Unterscheidung verstanden werden. Als eine solche bezieht sie sich einerseits auf die Differenz zwischen beobachtbaren Regelmäßigkeiten und nicht direkt beobachtbaren 'generativen' Regeln, gleichzeitig auf die Differenz zwischen dem, was unter spezifischen zeitlichen und räumlichen Bedingungen geschieht, und dem, was unabhängig vom zeitlichen und räumlichen Kontext existiert.

Soziale Praktiken sind selber keine Regeln, sondern Handlungsregelmäßigkeiten, beobachtbares repetitives Handeln. Regelcharakter haben hingegen die Wissensstrukturen: Die Regelmäßigkeit der Handlungsformen erscheint nur erklärbar, wenn man ihre Einbettung in jene Wissens-'Regeln' begreift, die von Bourdieu als Habitusforma- tionen, von Giddens als Regeln des practical consciousness eingeführt werden. ${ }^{6}$ Gleichzeitig kann man die kulturtheoretische Differenz zwischen Praxis und Struktur als eine Differenz zwischen dem Zeitkontingenten und dem Zeitresistenten sowie zwischen dem Kontextspezifischen und dem Kontextübergreifenden interpretieren. Soziale Praktiken finden zu bestimmbaren Zeitpunkten an bestimmten Orten, damit in einem jeweils einzigartigen Kontext statt. Die den Praktiken zugrundeliegenden Wissensstrukturen müssen hingegen als relativ zeitresistent und kontextunspezifisch gedacht werden: sie existieren über zeitliche und räumliche Grenzen hinweg (Giddens 1979: 59ff).

Als analytisch-heuristische Differenz bezieht sich die Unterscheidung von 'Wissensregeln' und 'Handlungssequenzen' jedoch nicht auf operativ voneinander unabhängig existierende Phänomene: Wissensstrukturen existieren immer nur insoweit, als sie tatsächlich in beobachtbaren Praktiken wirken und sich dort 'manifestieren'. Die Praktiken können von den Akteuren andererseits aber nur dadurch hervorgebracht werden, da $B$ diese über die entsprechenden Wissensbestände verfügen. Auch die Differenz zwischen der unterschiedlichen Zeitlichkeit und Kontextualität von Praxis und Struktur kann nicht ontologisch, sondern allein analytisch-heuristisch begründet sein: Wenn Wissensstrukturen nur in ihrer realen Anwendung in der Handlungssequenz existieren, muß ihre zeitliche Stabilität gegenüber dem Handlungsstrom ebenso relativiert werden wie die prinzipielle Kontextfreiheit der Regeln. Die Wissensbestände stellen dann nur in einem eingeschränkten Sinne einen 'Bestand' dar, da sie im zeit- und kontextspezifischen Handeln potentiell modifizierbar sind (Giddens 1979: 81ff).

Die Besonderheit der kulturtheoretischen Perspektive wird erst dann deutlich, wenn man ihren an der Leitdifferenz von Wissensstrukturen/ Regeln und Handlungspraxis ausgerichteten Theorierahmen als ein Vokabular interpretiert, das sich

\footnotetext{
${ }^{6}$ Hier ist nicht der Ort, auf die internen Unterschiede innerhalb des kulturtheoretischen Feldes einzugehen, die vor allem eine unterschiedliche Behandlung der Frage betrifft, wie der Akteur mit seinen inkorporierten Wissensstrukturen umzugehen vermag: Giddens etwa geht von der basalen Fähigkeit des Akteurs zu einem 'reflexive monitoring of action', einer Fähigkeit der Handlungssteuerung aus, die auch kontextspezifische Regelmodifikationen einschließt, während Bourdieu eine Neigung hat, in strukturalistischer Manier die Reproduktion der Wissensstrukturen 'durch den Handelnden' hindurch anzunehmen.
} 
jenseits der philosophisch und sozialtheoretisch wirkungsmächtigen Innen-Außen-Differenz von Subjekt und Objekt, von Bewußtsein und Welt, von Individuum und Gesellschaft bewegt. Drei Versionen einer solchen Innen-Außen-Differenz sind von besonderer Relevanz und gegen alle drei richtet sich das Vokabular der kulturtheoretischen Perspektive: die Subjektphilosophie bei Descartes und bei Husserl sowie das Paradigma des Homo sociologicus bei Durkheim.

Die Initialzündung zu einem ontologischen Deutungsschema, das um eine Innen-Außen-Differenz zwischen dem Bewußtsein einerseits, der 'Welt' außerhalb des Bewußtseins andererseits zentriert ist, läßt sich in Descartes' erkenntnisphilosophischen 'Meditationes' finden. Res cogitans und res extensa erscheinen hier als ontologisch differente Entitäten, die sich eindeutig voneinander abgrenzen lassen: das 'private', allein der Introspektion zugängliche, unteilbare Ich auf der einen Seite, die 'ausgedehnte' Objektewelt außerhalb des Bewußtseins, als Gegenstand der (Natur-)Wissenschaft auf der anderen Seite. (Descartes 1641)

Die Innen-Außen-Differenz zwischen Bewußtsein und Welt, die bei Descartes noch als ontologischer Dualismus gedacht ist, wird in Husserls Phänomenologie und in Durkheims Gesellschaftstheorie entontologisiert und in gegensätzlicher Form verarbeitet: In seiner Differenz zwischen ego cogito und cogitatum 'subjektiviert' Husserl die Außenwelt des Bewußtseins. Diese existiert nicht als eigenständige Entität, sondern allein als Korrelat der Bewußtseinsintentionen des Ego, das im zeitlichen Vollzug seiner 'cogitationes' die Welt als konstituiert durch einen spezifischen Sinnhorizont erfaßt. Diese Vorstellung setzt jedoch die Differenz zwischen Innen und Außen, zwischen, so Husserl, dem 'Eigenen' des Ego und dem 'Fremden', dem Nicht-Ego, zwischen Noetischem und Noematischem voraus. Die phänomenologische Epoché zwingt dazu, - vergleichbar Descartes' Methode des radikalen Zweifels - zunächst allein das 'reine Bewußtseinsleben' vorauszusetzen, um dann die Außenwelt des Bewußtseins als Phänomen, welches von dem Bewußtsein als solches erfaßt wird, von diesem different setzen zu müssen. (Husserl 1931)

Genau umgekehrt verfährt Durkheim in seiner Verarbeitung der Innen-Außen-Differenz. Hier wird nicht die Außenwelt subjektiviert, sondern in der Differenz zwischen Individuen und sozialen Tatsachen die Innenwelt objektiviert. Wenn Husserl Descartes' res cogitans zum Ausgangspunkt nimmt und die Welt damit als Korrelat dieses Bewußtseins deutet, so geht Durkheim gewissermaBen von der res extensa aus, verstanden als eine gesellschaftliche res extensa sozialer Tatsachen, die kausal auf die Subjekte und Bewußtseine einwirken. Die faits sociaux, die in erster Linie sanktionierte Normensysteme der Gesellschaft darstellen, üben 'von außen auf die Individuen Zwang aus', wirken damit als soziale Determinationskräfte von 'außen' nach 'innen'. Die für das Bild des Homo sociologicus typische Voraussetzung einer Externalität des Sozialen baut damit nur in einer anderen Version wiederum auf der prinzipiellen Unterscheidbarkeit zwischen einer subjektiven Innenwelt - hier: der individuellen 'Neigungen' - und der Außenwelt - hier: der gesellschaftlichen Normen - auf. (Durkheim 1895) ${ }^{7}$

So unterschiedlich und in manchen Hinsichten gegensätzlich die Perspektiven von Descartes, Husserl und Durkheim auch sein mögen, alle drei exemplifizieren damit das Denken in der InnenAußen-Differenz zwischen der Innenwelt eines 'eigenen' Ego und einer natürlich-körperlichen, intentional konstituierten oder sozialen 'fremden' Außenwelt.

Das Konzept einer Ermöglichung und Einschränkung der sozialen Praktiken durch kollektive, im-

7 Vgl. auch die Durkheim-Interpretation von Steven Lukes (1973: 16- 23), der zudem auf die Mehrdeutigkeiten im Durkheimschen Dualismus zwischen Gesellschaft und Individuum hinweist. Die Innen-Außen-Differenz zwischen Individuum und Gesellschaft wird dabei besonders deutlich in den 'Regeln der soziologischen Methode' formuliert, während sich Durkheims kultursoziologisches Spätwerk, das auf dem Begritf der 'représentations collectives' aufbaut, von diesem Dualismus löst. Daß Durkheims Homo sociologicus und Saussures Strukturalismus - der oben als Ahnherr der Kulturtheorien eingeordnet wurde im übrigen zwei parallele Fälle eines anti-individualistischen Denkens darstellen, das die Annahme einer emergenten Ebene des Sozialen eint, ist unbestritten. Der entscheidende Unterschied ist jedoch im jeweiligen Status dieser Emergenzebene zu sehen: Im Strukturalismus handelt es sich hier primär um eine kognitive Ordnung, die sich dem Individuum einschreibt und dieses dadurch erst konstituiert. Im Konzept des Homo sociologicus geht es hingegen primär um eine moralisch-normative Ordnung, der sich die Individuen - verstanden als neo-kantische homines duplices zwischen allgemeinen Pflichten und subjektiven Neigungen - gegenüberstehen. Wenn im Bild des Homo sociologicus die normative Ordnung wegfällt, bleiben immer noch die Individuen mit ihren subjektiven Neigungen übrig. Wenn im Strukturalismus die kognitive Ordnung wegfiele, verschwänden auch die Akteure, es bliebe - nichts. 
plizite und zugleich im einzelnen Akteur inkorporierten Wissensstrukturen in den Kulturtheorien ist nun von vornherein so gebaut, daß weder wie in der Subjektphilosophie 'das Bewußtsein' noch wie beim Homo sociologicus 'die sozialen Tatsachen' außerhalb des Bewußtseins und damit auch keine quasi-natürliche Differenz zwischen beiden als gegeben vorausgesetzt werden können. Wissensstrukturen werden einerseits als Regeln, die Sinnzuschreibungen anleiten, beschrieben, als überindividuelle, in diesem Sinne soziale Entitäten, die den jeweiligen 'subjektiven' Motiven und Handlungen immer schon vorausgehen und sich auch unabhängig vom einzelnen Subjekt analysieren lassen. Gleichzeitig können sie aber nur wirksam werden, indem sie von den Akteuren, die sie in ihr Handeln einfließen lassen, inkorporiert, implizit gewußt und im Handeln angewandt werden. Wissensgeleitete Praktiken lassen sich für die Kulturtheorien nur jenseits einer Differenz von privatem Bewußtsein und externer sozialer Welt begreifen; die Wissensregeln seien sozial, überindividuell und doch gleichzeitig 'im Akteur' vorhanden.

Pierre Bourdieu und Anthony Giddens argumentieren beide in ihren jeweiligen Fassungen einer 'Kulturtheorie' gegen das sozialtheoretische Denken in Innen-Außen-Differenzen. Ihre Argumentation wendet sich dabei einerseits gegen Versuche einer phänomenologischen Soziologie, ${ }^{8}$ andererseits und vor allem gegen das Bild des Homo sociologicus: Beide kritisieren an letzterem, daß hier 'das Soziale' als eine den Subjekten äußerliche Sphäre dargestellt wird, der nur deshalb in einem zweiten Schritt 'kausaler' Einfluß zugeschrieben werden kann, da sie von Anfang an dem Innern des Individuums gegenüber als different gedacht ist. Bei Bourdieu wird die Kritik am Homo sociologicus-Modell in eine Kritik am normativistischlegalistischen Denken in der Soziologie gekleidet, welches Handeln als Produkt externer, expliziter Normen statt inkorporierter, impliziter Habitusformationen deutet (1972: 203-227). Für Giddens handelt es sich in dieser 'strukturtheoretischen Soziologie' um eine Variante eines präjudizierten Dualismus zwischen Akteuren und sozialen Struk-

${ }^{8}$ Eine systematische kritische Auseinandersetzung mit Husserls Bewußtseinsphilosophie vor dem Hintergrund einer von Wittgenstein (allerdings kaum von Strukturalismus) beeinfluBten Sozialtheorie findet sich bekanntlich bei Habermas (1971). Zur Interpretation von Wittgenstein und von Saussure als zwei Versuche, die bewußtseinstheoretische Innen-Außen-Differenz zu überwinden vgl. auch Harland (1987: 123ff) und Rubinstein (1981: 95ff). turen „Explicitly or otherwise, such authors [das heißt Vertreter der an Durkheim angelehnten social facts-Soziologie] have tended to see in structural constraints a source of causation more or less equivalent to the operation of impersonal causal forces in nature...The structural properties of social systems, in other words, are like the walls of a room from which an individual cannot escape but inside which he or she is able to move around at whim." (Giddens 1984: 174; 1979: 49- 53).

Die Innen-Außen-Differenz muß aus der Perspektive der Kulturtheorien entweder in eine subjektivistische Soziologie oder in das Bild des Homo sociologicus oder in eine Kombination beider führen. In allen Fällen separiert sie eine Sphäre des Subjektiven und Psychischen von einer Sphäre des Sozialen: entweder muß dann der individuelle, dem Sozialen äußere Charakter der Subjekte behauptet werden oder aber der eigenständige, emergente Charakter des Sozialen jenseits und auBerhalb des Bewußtseins der Subjekte - letztlich zwei Positionen, die nur die Kehrseite der jeweils anderen darstellen. Die kulturtheoretische Konzeption wissensangeleiteter Praktiken geht hingegen immer schon davon aus, daß das, was scheinbar subjektive Besitzstände des einzelnen Bewußtseins sind, tatsächlich in übergreifende Regelstrukturen eingebettet ist, während umgekehrt die scheinbar eigenständigen, subjektunabhängigen sozialen Tatsachen nur bestehen, wenn sie in Form von Wissensstrukturen das reale Handeln der Akteure in deren 'Bewußtsein' (wenn auch 'vorbewußt') anleiten. ${ }^{\prime}$

\section{Die Innen-Außen-Differenz der Systemtheorie}

Die Ausgangsunterscheidung von Luhmanns konstruktivistischer Systemtheorie ist nicht die Differenz zwischen Wissensstruktur und Handlungspraxis, sondern die zwischen System und Umwelt, zwischen einem sich selbst von seiner Umwelt dif-

${ }^{9}$ Zygmunt Bauman formuliert in ähnlicher Richtung: Kultur „transcend(s) the opposition between the subjective and the objective.... It is, simultaneously, the objective foundation of the subjectively meaningful experience and the subjective 'appropriation' of the otherwise inhumanly alien world.... It resists stubbornly all attempts to associate it unilaterally with either one or the second pole of the experimental frame. The concept of culture is subjectively objectified; it is an effort to understand how an individual action can possess a supra-individual validity (in: Bauman 1973: 116f). 
ferent setzenden System - das erst durch die Differentsetzung seine Identität erlangt - und eben dieser Umwelt. ${ }^{10}$ Dieses Schema der sich selbst voneinander different setzenden Systeme läßt sich auf verschiedenartige Systemtypen anwenden, besonders folgenreich wird die System-Umwelt-Unterscheidung jedoch in der Differenz zwischen den beiden verschiedenartigen Typen von Sinnsystemen, den psychischen und den sozialen Systemen.

Warum soll man nach Luhmann dem Imperativ 'draw a distinction' nun aber genau so und nicht anders folgen und zwischen sozialen und psychischen Systemen unterscheiden? Solange man Systemtheorie betreibt, stellt sich diese Differenz ebenso wie die zwischen einzelnen psychischen und zwischen einzelnen sozialen Systemen - nicht als eine 'analytische', 'lediglich' heuristisch motivierte Theorieentscheidung dar, sondern ist 'durch den Gegenstand selber aufgezwungen'. Die Frage, ob ein System vorliegt oder nicht, fällt, so Luhmann, nicht in den Ermessensspielraum des wissenschaftlichen Beobachters, sondern ist in der beobachteten 'Realität' bereits immer schon beantwortet: ${ }^{11}$ Die operativen Beobachtungen der sinnhaften Realität - die die Sozialwissenschaft nun ihrerseits beobachtet - setzen sich nämlich selber bereits von anderen Operationen und Beobachtungen different und legen damit fest, wo die eigenen und wo die fremden Operationen und Beobachtungen verlaufen, bestimmen mithin, was zum eigenen System gehört und was zur Umwelt dieses Systems (Luhmann 1988: 38; 1990: 65; 1992b: 28).

Entscheidend für die systemtheoretische Voraussetzung einer Differenz von System und Umwelt

${ }^{10}$ Auf diesen Prozeß des systemischen Sich-von-derUmwelt-Differentsetzens, der erst die eigene Identität ermöglicht, wird verwiesen, wenn Luhmann von der 'Differenz zwischen Identităt und Differenz' der neueren, beobachtungstheoretischen Systemtheorie spricht (1984: 26). Freilich baut diese Differenz weiterhin auf der 'älteren' System-Umwelt-Differenz auf. Wagner/ Zipprian (1992) weisen daher zurecht darauf hin, daß letztlich bei Luhmann immer eine Identität vorausgesetzt bleiben muß: die der beobachtenden Operation selber.

11 „Das Belieben des Beobachters liegt in der Wahl des Systems, von dem er ausgeht, nicht aber in der Frage, was er als System behandeln kann." (Luhmann 1990: 65). Gelegentlich klingt Luhmanns Begründung für die Wahl des systemtheoretischen Unterscheidungsschemas dabei erstaunlich 'realistisch': „...muß man bei einer theoretischen Erklärung, die sich auf die wirklichen Verhältnisse einläßt, Bewußtseinssysteme und kommunikative Systeme (soziale Systeme) streng unterscheiden." (1988: 39, Hervorhebung A.R.) ist somit die Voraussetzung der Existenz von Operationen, von Ketten ereignishafter Elemente gleichen Typs, die sich 'autopoietisch' aus sich selber produzieren. Sofern in diesen Operationen Sinn prozessiert wird, handelt es sich gleichzeitig um 'Beobachtungen', Beobachtungen der Umwelt oder des eigenen Systems, die in der Form von Bezeichnungen auf der Grundlage sinnhafter Unterscheidungen verlaufen. In Luhmanns 'operativem Konstruktivismus' bildet sich damit das, was man ein 'System' nennen kann, dadurch, daß Beobachtungsoperationen sich von fremden Operationen und Beobachtungen selber unterscheiden und sich durch diese Unterscheidung als eigene Beobachtungsoperationen $z u$ identifizieren vermögen.

Ein psychisches System ist daher ein psychisches System, nicht weil der wissenschaftliche Beobachter es auf diese Weise identifiziert, sondern weil es sich selber als Kette von Gedanken von 'allem anderen', das heißt von fremden, unzugänglich erscheinenden Ketten von Gedanken - also anderen psychischen Systemen ('ich im Unterschied zu den anderen') - sowie von ihnen 'äußeren' Kommunikationen - die im Unterschied zu den privaten Gedanken öffentlich stattfinden ('mein Denken im Unterschied zum Reden') - unterscheidet und damit eine eigene 'Identität' als 'Bewußtsein' erlangt. Analoges gilt für ein soziales System: Ein soziales System ist ein soziales System, indem sich eine Kette von Kommunikationen selber von allem anderen außer ihr unterscheidet - von Kommunikationen anderen Typs, denen andere Erwartungsstrukturen und Semantiken zugrundeliegen, und erst recht von den 'unzugänglichen' privaten $\mathrm{Ge}$ danken innerhalb der Bewußtseine - und sich damit als soziales System zu identifizieren weiß. Psychische Systeme/ Bewußtseine und soziale Systeme/ Kommunikationen konstituieren sich jeweils dadurch als ein 'Selbst', daß sie sich von allem Fremden, Äußeren different setzen. Luhmann verwendet dabei selber den Begriff der 'InnenAußen-Differenz' (1996: 33): Das System identifiziert sich selbst als ein Innen dadurch, daß es sich von einem $A u ß e n$ sinnhaft unterscheidet.

Luhmanns auf der Differenz zwischen System und Umwelt beruhender Konstruktivismus erweist sich damit - vielleicht überraschenderweise - in einer neuartigen Form als 'teilnehmerorientierte' Soziologie. Im Gegensatz etwa zur Systemtheorie Parsons', die ihre strikt 'analytischen' Unterscheidungen zwischen verschiedenen Systemtypen allein dem wissenschaftlichen Beobachter schuldet, will Luhmann die Differenz zwischen psychischen und sozialen Systemen (sowie zwischen einzelnen 
psychischen und zwischen einzelnen sozialen Systemen) allein dadurch gewinnen, daß sich der Sozialwissenschaftler den entsprechenden Differentsetzungen der 'Teilnehmer' selber anschließt: Die Teilnehmer sind hier nur nicht wie in der handlungstheoretischen Soziologie die 'Akteure', sondern die Bewußtseine und die Kommunikationen, die in der sinnhaften Welt bereits ohne Zutun des Sozialwissenschaftlers operieren. ${ }^{12}$

Luhmanns Innen-Außen-Differenz zwischen psychischen und sozialen Systemen sowie zwischen einzelnen psychischen Systemen greift auf zwei Argumentationsquellen zurück: einerseits auf die des Alltagsverstandes, das heißt der Selbst- und Fremdbeschreibungen der 'psychischen Systeme', andererseits auf die der Husserl'schen Bewußtseinsphilosophie. Luhmann appelliert regelmäßig stillschweigend an den 'Alltagsverstand' (selbstverständlich ohne diesen Begriff zu gebrauchen), stützt sich auf Deutungsschemata der 'Lebenswelt des Alltags', um sich der 'Selbstverständlichkeit' der immer schon vorausgesetzten Innen-AußenDifferenz zu versichern, die drei Aspekte umfaßt:

Erstens muß man 'natürlicherweise' eine Differenz zwischen 'meinem Ich' und 'allem anderen' außerhalb dieses Ichs voraussetzen. Dieses 'alles Andere' umfaßt zunächst die fremden Bewußtseine, die als black box prinzipiell unzugänglich bleiben, so wie meine Gedanken als privater Besitz anderen Ichs gleichfalls unzugänglich sind: „Bewußtsein ist intern - und zum Glück ist es intern, es wäre ja schrecklich, wenn jeder in das Bewußtsein eines anderen einsteigen und dort hineindenken könnte“ (1992c: Vorlesung 4) „Ich selbst jedenfalls würde mich in der Umwelt der Gesellschaft wohler fühlen als in der Gesellschaft, wo ... andere Leute meine Gedanken denken." (1992c: Vorlesung 11)

Zweitens gilt für den Alltagsverstand wie selbstverständlich: Wenn die Bewußtseine einander per definitionem fremd sind, jedes für sich operiert, dann müssen sie allesamt verschieden sein. Mit Gewißheit kann man damit nur Aussagen über das eigene machen. Jedes dieser Bewußtseine sieht die Welt anders, das heißt, jedes benutzt individuell

12 Insofern kann es nicht schlüssig erscheinen, wenn etwa Habermas Luhmann ,nicht etwa nur objektivierende, sondern objektivistische Beschreibungen" (Habermas 1985: 444) vorwirft. Objektivistisch wäre eine Beschreibung, die (allein) selbstgewählte Unterscheidungen verwendet, aber nicht eine, die an Unterscheidungen anschließt, die in der sinnhaften Welt selber vorkommen (zumindest dem Urteil des Beschreibenden nach). verschiedene Beobachtungsschemata: „Würde man für ein psychisches System optieren, stünde man vor der Wahl: welches von den etwa fünf Milliarden? Und die Entscheidung könnte dann praktisch nur lauten: ich selber." (1990: 63).

Drittens umfaßt die Umwelt meines Ichs auch die Sphäre der Kommunikationen: Im Alltagsverstand wird natürlicherweise zwischen Denken und Reden unterschieden. Das Reden erscheint hier als öffentlich, das Denken als privat. Der Alltagsverstand weiß, daß Reden und Denken voneinander differieren können; was gesprochen wird und was jemand denkt, muß nicht übereinstimmen: „Ein Arzt tritt ans Krankenbett und fragt: wie geht es Ihnen? Wie peinlich, wenn man dabei etwas über das erfahren würde, was er sich dabei denkt." (1992d: 140). Die Gedankenkette, die man Bewußtsein nennt, setzt sich also selber von der Kommunikation 'außerhalb ihrer selbst' different. Insgesamt gilt somit: Die Unterscheidung zwischen einzelnen Bewußtseinen sowie zwischen Bewußtsein und Kommunikation als zwei Operationstypen ist gerade keine kontraintuitive, 'verfremdende', sondern schließt an das gewohnte Denken alltäglicher Deutungsschemata an.

Philosophiehistorisch greift Luhmanns InnenAußen-Differenz zwischen System und Umwelt sowohl mit Blick auf psychische als auch - im übertragenen Sinne - mit Blick auf soziale Systeme ausdrücklich auf Husserls Phänomenologie zurück $(1994,1996):{ }^{13}$ Für Luhmann läßt sich diese als Beispiel einer allgemeinen Theorie der Selbstproduktion von Innen-Außen-Differenzen neubeschreiben, das freilich noch allein auf die Systemreferenz Bewußtsein bezogen bleibt. Das intentionale Bewußtsein Husserls ist für Luhmann nichts anderes als ein autopoietisch-selbstreferentielles System, welches sich durch das Setzen einer Unterscheidung (die dann entsprechende Bezeichnungen anleitet) von seiner Umwelt different setzt und sich damit als Bewußtsein konstituiert. Husserls Bewußtsein beobachtet wie Luhmanns System seine Umwelt gemäß eigener Unterscheidungsschemata und produziert sich damit - auf der Grundlage dieser mitlaufenden Selbstreferenz selber. Husserl unterscheidet allerdings noch eine Ebene des Empirischen von der des Transzendentalen - für Luhmann eine unnötige begriffliche

${ }^{13}$ Habermas hat bekanntlich schon fruh und in äußrst klarer Weise auf Luhmanns Verarbeitung von Husserls Subjektphilosophie hingewiesen (1985: 426- 445). Vgl. auch Englisch 1991 und Ellrich 1992. Mittlerweile hebt Luhmann ausdrücklich diese 'Erbschaft' hervor. 
Verdopplung - und bezieht vor allem den Kerngedanken der Selbstproduktion von Innen-AußenDifferenzen allein auf das Bewußtsein. Luhmann überträgt ihn auch auf soziale Systeme, auf Kommunikationen, beläßt ihn aber mit Blick auf Bewußtseine, auf psychische Systeme unverändert. Die Systemtheorie „verwendet, wie die Bewußtseinsphilosophie, das Konzept der Geschlossenheit der selbstreferentiellen Reproduktion des Systems.“ (1986b: 171) „Für den Fall von Bewußtseinsleistungen könnte man Husserls Analysen wiederholen... Für den Fall von Kommunikationen müßte man eine Parallelkonstruktion finden, die auch hier das nachweist, was, und es so nachweist, wie es im Falle des Bewußtseins funktioniert." (1996: 50).

Luhmanns Innen-Außen-Differenz zwischen psychischen und sozialen Systemen hat nun entscheidende Konsequenzen für seine Konzeptualisierung von Sinnmustern. Grundsätzlich ist das Medium 'Sinn' den sozialen und psychischen Systemen gemeinsam: Wenn sich Sinn als ein „Prozessieren nach Maßgabe von Differenzen" (1984: 101) verstehen läßt, so stellen sich Kommunikationen wie Gedanken als sinnhafte Beobachtungen, als Sinnsysteme dar. Aber das kann für das operationalistische Denken gerade nicht bedeuten, da $\beta$ Sinn gewissermaßen 'grenzüberschreitend' beide Systemtypen als eine gemeinsame Struktur überwölbt. Die Innen-Außen-Differenz zwingt vielmehr zu einer Bifurkation von zwei irreduziblen Typen von Sinnstrukturen, die die jeweiligen Beobachtungsoperationen anleiten: Auf der einen Seite existieren jene Unterscheidungsschemata, die den Beobachtungen eines psychischen Systems zugrundeliegen, auf der anderen Seite jene, die die Beobachtungen eines sozialen Systems anleiten. Nur diejenigen Sinnstrukturen, die den Kommunikationen, den sozialen Systemen, zugrundeliegen, belegt Luhmann dabei ausdrücklich mit dem Begriff des 'Wissens' (1990: 133), während er sich für psychische Systeme mit dem allgemeineren Begriff der 'Erwartungen', vor allem in Form von 'Ansprüchen' behilft.

Was ist nun 'Wissen' als die Sinnstruktur von sozialen Operationen? Sobald in einem sozialen System Sinn 'kondensiert', mithin auf Dauer gestellt und 'konfirmiert' wird, das heißt, in verschiedensten Situationen anwendbar ist, sobald sich damit relativ stabile Unterscheidungsschemata ausgebildet haben, kann man vom 'Wissen' eines sozialen Systems sprechen: „Nichts anderes ist gemeint, wenn wir gelegentlich von Semantik sprechen" (1990: 107). Wissen als soziale Sinnstruktur ist mit Se- mantik identisch. Wenn soziale Systeme nichts anderes sind als Kommunikationen, so müssen die stabilisierten Unterscheidungsschemata, so muß das soziale Wissen eine sprachliche Form besitzen (zumindest soweit Kommunikation sprachlich ist) und somit als 'Semantik' auftreten, womit keinesfalls allein die 'gepflegte Semantik' der Schriftsprache gemeint ist, sondern jegliche kommunikationsstrukturierende Semantik. (1990: 107f, Kap. 1-3) $)^{14}$

Wie sich die eigenständigen Beobachtungen der psychischen Systeme beschreiben lassen, ist für eine Theorie sozialer Systeme umgekehrt nur ein marginales Problem, vielmehr für Luhmann primär die Aufgabe der Psychologie. Er legt lediglich fest, daß psychische Systeme sich als Operationstypus 'Gedanken' identifizieren lassen, die, sobald sie ihrerseits (fremd- oder selbst-) beobachtet werden, die Form von 'Vorstellungen' annehmen. Die Unterscheidungen und Bezeichnungen der psychischen Systeme erlauben auch diesen - ähnlich den sozialen Systemen -, kognitive 'Erwartungen' an ihre Umwelt auszubilden, die sich insbesondere an bestimmtes 'Verhalten' innerhalb von sozialen Systemen richten, Erwartungen, die sich dann zu kontrafaktisch stabilisierten 'Ansprüchen' verdichten. (1984: Kap. 7; 1987b)

Luhmann stellt fest, daß das einzelne psychische System - und im übertragenen Sinne auch das einzelne soziale System - sich jeweils durch 'Individualität' auszeichnen. Mit dieser Beschreibung schließt er an die von ihm selber in anderem Kontext rekonstruierte typisch moderne Semantik des Individuums und der Individualität positiv an (Luhmann 1987c; 1989: 149- 258). 'Individualität' als semantisches Konzept, das sich ursprünglich in den ästhetischen, philosophischen und politischen Debatten um Originalität, Bewußtsein und Individualismus im 18. und 19. Jahrhundert ausgebildet hat, läßt sich in dem Grundsatz zusammenfassen: „Das Individuum ist die Welt im besonderen Ich" (1989: 207) ,...Individualität ist, um eine glückliche Formulierung von Georg Simmel zu zitieren, die Normung des Ich durch das Ich." (1989: 213) und genau diese Aussage läßt sich im Kern nun auf die psychischen Systeme allgemein übertragen.

${ }^{14}$ Der Wissensbegriff wird dabei von Luhmann in ähnlicher Weise wie von Giddens temporalisiert: Strenggenommen kann es keine überzeitlichen Wissensbestände geben, real ist vielmehr lediglich das Wissen, das in der operativen Sequenz bzw. in der Sequenz sozialer Praktiken angewandt wird (Luhmann 1990: 128ff; Giddens 1979: 198ff). 
Wenn für Luhmann psychische Systeme 'individuell' sind, es zur Zeit von ihnen - wie er gerne betont - 'sechs Milliarden verschiedene gibt', so heißt dies nichts anderes, als daß jedes Bewußtsein gemäß seinem eigenen, individuellen Beobachtungsschema operiert: „Daher sieht für jedes psychische System die Welt anders aus." (1991: 145). Wenn man diese Einsicht nicht nur auf psychische, sondern auch auf soziale Systeme bezieht, kann Luhmann seine Theorie berechtigterweise als einen 'radikal individualistischen' Ansatz beschreiben: „die Theorie autopoietischer, sich-selbstausdifferenzierender Systeme (ist) eine radikal individualistische Theorie ..., weil sie ihre Individuen ... durch jeweils eigene, selbstkonstituierte Umweltperspektiven, also durch jeweils anders konstruierte Welteinschnitte kennzeichnet." (1994: 165).

Die Bifurkation zwischen den psychischen und den sozialen Systeme auf der Ebene ihrer Operationen, Beobachtungen und Schemata der Beobachtung bedeutet auch für die Systemtheorie nun bekanntlich nicht, daß beide beziehungslos nebeneinander existierten. Freilich muß das Verhältnis der Systeme zueinander so konzeptualisiert werden, daß die operative Differenz zwischen beobachtenden Systemen und ihrer Umwelt erhalten bleibt. Dies soll der Begriff der 'strukturellen Kopplung' leisten. Strukturelle Kopplungen können für Luhmann im Rahmen einer Theorie operativ geschlossener Systeme keine Kausalitätsbeziehung bezeichnen. Die Annahme von intersystemischen Ursache-Wirkungs-Beziehungen würde die Systeme als Trivialmaschinen mißverstehen, die ein Input mit einem Output beantworten. Wenn jedes operative Sinnsystem, ob psychisch oder sozial, nur entlang der eigenen Differenzschemata zu beobachten vermag, so kann es unmöglich von außen determiniert, sondern allein durch Ereignisse in seiner Umwelt 'irritiert' werden. Irritiert werden soziale Systeme nun regelmäBig durch Ereignisse der psychischen Systeme sowie möglicherweise durch bestimmte andere soziale Systeme in ihrer Umwelt, psychische Systeme wiederum erfahren regelmäßige Irritationen durch Ereignisse der Kommunikationen sozialer Systeme. ${ }^{15}$ 'Irritationen' können per definitionem immer nur auf der Grundlage der eigenen systemischen Beobachtungsschemata wirken. Strukturelle

${ }^{15}$ Luhmann weist in diesem Zusammenhang im übrigen darauf hin, daß 'Sprache' das wichtigste Medium der strukturellen Kopplung zwischen sozialen und psychischen Sinnsystemen ist (1990: $47 \mathrm{ff}$ ).
Kopplungen sind somit nichts anders als - allein durch einen externen Beobachter feststellbare (1990: 530 - hier muß Luhmann seine Teilnehmerperspektive verlassen) - regelmäßige Irritationsverhältnisse zwischen verschiedenen Operationssequenzen, Irritation bedeutet für Luhmann Konfrontation eines Systems mit Ereignissen aus seiner Systemumwelt, und zwar eine Konfrontation nach Maßgabe der Unterscheidungsschemata des eigenen Systembildschirms (1990: 38ff, 163ff). ${ }^{16}$ Das Konzept der strukturellen Kopplung bestätigt damit die prinzipielle Bifurkation zwischen den Operationen des Psychischen und denen des Sozialen.

Betrachtet man Luhmanns konstruktivistische Systemtheorie vor dem Hintergrund der Kontrastfolie der 'Kulturtheorien', so kommt man zu folgendem Schluß: Während die Kulturtheorien jenseits der Innen-Außen-Differenz zwischen Bewußtsein und (sozialer) Welt formuliert werden, vielmehr auf der analytischen Unterscheidung von Regeln und Handeln aufbauen und damit handlungsanleitende Wissensstrukturen als sozial emergent und subjektiv wirksam gleichermaßen annehmen, baut Luhmann umgekehrt mit expliziter Berufung auf Husserl auf dieser Innen-Außen-Differenz in der flexibilisierten Form einer Theorie autopoietischselbstreferentieller Systeme auf. Die Sphäre des Psychischen und die Sphäre des Sozialen verlaufen in diesem Theorierahmen voneinander separiert und stellen hier als psychische Systeme und als soziale Systeme operational getrennte Sinnsequenzen dar.

Luhmanns Bifurkation zwischen sozialen und psychischen Systemen läßt sich theoriehistorisch als eine innovative Kombination der leitenden InnenAußen-Differenzen von Husserl und von Durkheim lesen. Von Husserl entlehnt Luhmann den Gedanken der sinnhaften Selbstproduktion von Innen-Außen-Differenzen, von Differenzen zwi-

16 Vom Begriff der strukturellen Kopplung zu unterscheiden ist der neuerdings von Luhmann gelegentlich für intersystemische Beziehungen verwendete Begriff des 'Materialitätskontinuums' $(1990: 30,39)$. Während der Begriff 'strukturelle Kopplung' 'lediglich' Irritationsverhältnisse bezeichnen kann, soll der Begriff 'Materialitätskontinuum' offenbar auf 'tieferliegende' gegenseitige Konstitutionsbeziehungen verweisen (die früher der Begriff der Interpenetration mitabgedeckt hatte, vgl. 1984: Kap. Kap.6), darauf, daB System $X$ auf die Existenz eines Systems $Y$ 'angewiesen' ist. Diesen Zusammenhang in einer operationstheoretisch fundierten Theorie präzise auszudrücken, scheint jedoch beträchtliche Schwierigkeiten zu bereiten. 
schen 'eigenen' und 'fremden' Operationen. Husserl formuliert jedoch eine derartige Differenz allgemein zwischen Bewußtsein und 'Welt' und nicht im speziellen eine Differenz zwischen Bewußtsein und Sozialwelt. Damit wird vorausgesetzt, daß das Selbst, das seine Identität durch Differentsetzung zur 'Außenwelt' gewinnt, immer nur das Bewußtsein und nicht die 'Welt' außerhalb des Bewußtseins sein kann. $\mathrm{Da}$ sich umgekehrt die Welt von den Bewußtseinen different setzen könnte, wäre undenkbar.

Für Luhmanns Innen-Außen-Differenz ist nun aber gerade die Differenz zwischen Bewußtsein und Sozialwelt zentral - und diese kann er von Durkheim übernehmen. Durkheims theoriekonstitutive Innen-Außen-Differenz ist die zwischen Individuum und Gesellschaft, zwischen Individuen und sozialen Tatsachen. Für Luhmann ist wie für Durkheim die Sozialwelt gegenüber der Innenwelt der Individuen 'extern'. Umgekehrt sind dann auch die Individuen gegenüber der Sozialwelt extern. Damit wird für Durkheim wie für Luhmann jener Wechsel der Systemreferenz vollzogen, der für die Soziologie entscheidend ist: das Innen braucht nicht mehr zwangsläufig das Bewußtsein, sondern kann auch die soziale Welt sein. Freilich bleibt die Innen-AuBen-Differenz bei Durkheim dem Gedanken einer vorkonstruktivistischen System-Umwelt-Differenz verhaftet, die die selbstreferentielle Wende noch nicht vollzogen hat, vielmehr System und Umwelt als gegebene Entitäten mit Input-Output-Beziehungen statt als sich sinnhaft selbstproduzierende Operationen konzeptualisiert.

Das Ergebnis von Luhmanns Kombination von Husserls Differenz zwischen Bewußtsein und Welt und Durkheims Differenz zwischen Individuum und Gesellschaft ist eine gegenüber Husserl flexibilisierte Form der Innen-Außen-Differenz. Was das 'Innen' ist, das sich von einem 'Außen' different setzt, erweist sich nun als variabel: die Systemreferenz kann sich auf das psychische System genausogut wie auf das soziale System beziehen. Die bewußtseinsphilosophische Sackgasse, in die Husserl geraten war und die ihm die Konzeptualisierung des Sozialen verunmöglichte (Schütz 1957; Habermas 1971), wird von Luhmann mithin gleichfalls erkannt, aber radikal anders aufgelöst als in den Kulturtheorien und ihren philosophischen Vorläufern bei Saussure und Wittgenstein. Während letztere sich jenseits des Denkmusters der Innen-Außen-Differenz zwischen Bewußtsein und Außenwelt situieren, schließt Luhmann ausdrücklich an diese Differenz an - und versucht das Problem gewissermaßen durch eine 'Verdopplung' der relevanten Sinnsysteme zu lösen: Den gegenüber der Phänomenologie unverändert gedachten Bewußtseinen wird ein ähnlich gebauter Typus 'Kommunikationssysteme' gegenübergestellt, der schon per definitionem aus einem genuin sozialen, den Bewußtseinen theoretisch analog gebauten Operationstyp besteht. Wenn das BewuBtsein als autopoietisch-selbstreferentielles System beschrieben wird und weiterhin beschrieben werden soll (aus dem dann das Soziale konsequenterweise nicht abgeleitet werden kann) und trotzdem die Vorstellung einer emergenten Ebene des Sozialen $\mathrm{zu}$ verteidigen ist, dann bleibt als theoriearchitektonisch konsequente Lösung, das Soziale wie das Bewußtsein als eigenständigen Typus einer autopoietisch-selbstreferentiellen Operation zu denken. Mit der Innen-Außen-Differenz zwischen Psychischem und Sozialem scheint es Luhmann damit möglich, 'to have the cake and to eat it': Bewußtseine nach Art der Bewußtseinsphilosophie $\mathrm{zu}$ verstehen - und gleichzeitig begrifflich über eine genuine Sphäre des Sozialen zu verfügen.

\section{Verschobene Fronten: Kulturtheoretischer Holismus, systemtheoretischer Individualismus und die Frage des Wissensbegriffs}

Es ist deutlich geworden, da $\beta$ trotz ihrer grundsätzlich gemeinsamen Einsicht in die sinnhafte Konstitution der Wirklichkeit Luhmanns Systemtheorie und die Kulturtheorien völlig verschiedenen Aufbauprinzipien gehorchen: Im Kern von Luhmanns Ansatz steht die Innen-Außen-Differenz zwischen Sozialem und Psychischem. Die Kulturtheorien von Bourdieu und Giddens stehen, dem Strukturalismus und Wittgenstein folgend, diesem Denken in Innen-Außen-Differenzen fern. Luhmann beschreibt deshalb in Form der InnenAußen-Differenz - die sich auch auf die Beziehung zwischen mehreren sozialen oder mehreren psychischen Systemen untereinander beziehen läßt -, weil seine Theorie operationstheoretisch ausgerichtet ist: So soll sich 'wie natürlich' die Differenz zwischen psychischen Operationen/Gedanken und sozialen Operationen/Kommunikationen ergeben, die sich bereits selber in ihrer Beobachtung voneinander different setzen. Die Praxistheorien übernehmen demgegenüber nicht die Unterscheidungen, mit denen sich 'Bewußtseine' oder 'Kommunikationen' selber voneinander differieren, sondern markieren eine analytische Differenz zwischen Struktur und Praxis. 
Die unterschiedlichen Theorieentscheidungen in Luhmanns Systemtheorie und den Praxistheorien wirkt sich darauf aus, wo jeweils 'Sinnmuster' und wo 'das Soziale' zu verorten ist. 'Sinn' muß für Luhmann zwangsläufig den Operationen (die dann gleichzeitig Beobachtungen sind) zugerechnet werden. Da hierbei jedoch zwei verschiedene Operations- und damit Systemtypen als mögliche Kandidaten in Frage kommen, Kommunikationen und Gedanken, so ist eine Bifurkation von Sinnsequenzen und damit Sinnmustern nötig: Dem in der Kommunikation prozessierten Sinn liegen - trotz aller struktureller Kopplungen - zwangsläufig andere Beobachtungsschemata zugrunde als dem in Gedanken prozessierten Sinn. Aus der Sicht der Kulturtheorien hingegen besteht kein Grund, von zwei verschiedenen Sinnstrukturen - die eine im Bewußtsein, die andere in der sozialen Welt - auszugehen: Sinnzusammenhänge liegen vielmehr den sozialen Praktiken als Wissensstruktur zugrunde. Die Sinnzusammenhänge müssen also gewissermaßen einer Struktur von sozialen Deutungsregeln und den Akteuren zugleich zugerechnet werden, die diese in ihrem Handeln verwenden.

Entsprechend ergeben sich auch zwei unterschiedliche Verortungspunkte des Sozialen: Das Soziale muß für Luhmann eine Operation sein. Dafür kommt nur Kommunikation in Frage, da allein die sprachliche Sequenz der Kommunikation eine immanent strukturierte und in diesem Sinne von subjektiven Intentionen unabhängig existierende, sich selber reproduzierende Sinnebene darstelle. ${ }^{17}$ Folgt man dem Begriff des Bewußtseins der Subjektphilosophie und der dortigen Unmöglichkeit, eine Kollektivität von Bewußtseinen zu denken, können hingegen psychische Systeme selber nicht der Ort des Sozialen sein.

Aus der Sicht der Kulturtheorien ist das Soziale demgegenüber in den als Wissen inkorporierten und von den Akteuren angewandten Regeln festzumachen. Denn diese Wissensregeln sind es, die dafür verantwortlich zeichnen, daß Handeln nicht beliebig und individuell sowie kontextspezifisch

${ }^{17}$ Es kann an dieser Stelle nicht darum gehen, zu problematisieren, ob und inwiefern es Luhmann tatsächlich gelungen ist, 'Kommunikation' als eigenständige, autopoietische Operation zu beschreiben oder ob sich die angenommenen Selektionselemente der Kommunikation (Mitteilung, Information und Verstehen) nicht vielmehr nur mit Bezug auf Akteure oder Bewußtseine nachvollziehen lassen (vgl. die entsprechende Kritik von Roth 1987 und Martens 1991). einzigartig verläuft, sondern innerhalb von sozialen Kollektiven in verhältnismäßig regelmäßigen Mustern. Die klassische i.e.S. 'handlungstheoretische' Unterscheidung zwischen 'Handeln' und 'sozialem Handeln' verliert damit für die Kulturtheorien an Bedeutung: Indem Handeln auf der Grundlage kollektiver Wissensregeln stattfindet, hat es unweigerlich ein soziales Fundament. $\mathrm{Ob}$ Handeln subjektiv sozial orientiert ist oder nicht, stellt sich damit für die Kulturtheorien als eine verfehlte Frage dar. Die sinnhaften Regelstrukturen, die den Praktiken zugrundeliegen, sind nämlich zweifellos bereits sozial konstituiert - und wirken sich damit selbst auf das Handeln eines Robinson aus, dessen Handeln keine intentionale soziale Orientierung' besitzt.

Wenn man sich die Theoriebauprinzipien des systemtheoretischen Konstruktivismus vor dem Hintergrund der Theoriearchitektur der Kulturtheorien vergegenwärtigt, lassen sich gängige Linien der gegenseitigen Theoriekritik nicht aufrechterhalten: Die Kritik Luhmanns an seinen 'handlungstheoretischen' Konkurrenten läuft auf das Urteil hinaus, es handele sich hier um einen Methodologischen Individualismus, der Soziales nicht wirklich zu konzeptualisieren vermag. Die gängige Kritik mancher Handlungstheoretiker an Luhmanns Systemtheorie lautete in der Vergangenheit umgekehrt, hier liege ein sozialer Holismus vor, der die Individuen, Akteure, Subjekte dadurch marginalisiere, daß er diese in die Umwelt des Sozialen 'abschiebe'.

Luhmann setzt seine Systemtheorie bekanntlich von der Tradition der 'Handlungstheorie' ab. Mit den 'Theorien der Praxis' von Bourdieu und Giddens, auch mit Wittgenstein und Saussure ${ }^{18}$ mithin mit den Autoren die hier für eine Kulturtheorie bedeutsam erscheinen, findet zwar keine ausdrückliche kritische Auseinandersetzung statt, es fällt aber nicht schwer, auch auf diese Ansätze Luhmanns zentrales Argument gegen die Handlungstheorien zu beziehen: Luhmann wirft diesen vor, keinen Begriff des Sozialen jenseits des Begriff des Subjekts zu besitzen. Das Soziale werde letztlich in Beziehungen zwischen Subjekten, in der 'Intersubjektivität' festgemacht, die sich aber am Ende immer nur über die bereits vorausgesetz-

${ }^{18}$ Gelegentlich bezieht sich Luhmann jedoch zustimmend auf Saussures Semiotik als Begründung eines 'Unterscheidungsrelativismus' (1990: 99), setzt sich jedoch an anderer Stelle vom strukturalistischen Kerngedanken einer Generierung von 'Operationen' aus Regeln deutlich ab (1987b: 61). 
ten Subjekte definieren lasse, so daß hier niemals eine emergente Ebene des Sozialen erreicht werde und letztlich immer eine Spielart des Methodologischen Individualismus übrigbleibe. (Luhmann 1986b; 1990: 68- 72; 1995a: 153- 162)

Es wird nun deutlich, wie dieser Einwand eigentlich zu verstehen ist und daß er sich auf die Kulturtheorien kaum beziehen läßt. Luhmanns Kritik an den Handlungstheorien setzt die Gültigkeit der Innen-Außen-Differenz zwischen Bewußtsein und sozialer Welt immer schon voraus. Für Luhmann müssen die Handlungstheorien in ihrem Begriff des Sozialen dann offenbar in der gleichen Weise scheitern, wie Husserls Bewußtseinsphilosophie in der Konzeptualisierung der Sozialität im Begriff der 'Intersubjektivität' und der 'Monadengemeinschaft' - nach gängigem und auch nach Luhmanns eigenem Urteil (Schütz 1957; Luhmann 1994) scheiterte. Die Systemtheorie geht selber von der Innen-Außen-Gedankenfigur der Subjektphilosophie aus (gibt dieser freilich mit dem Begriff des außerpsychischen sozialen Systems eine Wendung) - und kann vor dem Hintergrund dieses innen-außen-theoretischen Deutungsschemas offenbar nicht anders, als in die Handlungstheorien hineinzulesen, ebenfalls an die Subjektphilosophie anzuschließen. Für Luhmann muß es sich bei den Handlungstheorien um Versionen der Bewußtseinstheorie traditioneller Form handeln, in der die Systemreferenz auf psychische Systeme festgelegt ist und soziale Systeme als eigenständige Operationsebene noch nicht erkannt werden.

Um auf der immer schon vorausgesetzten Grundlage der Innen-Außen-Differenz 'das Soziale' zu erfassen, scheint es für Luhmann immer nur zwei Möglichkeiten zu geben: Entweder man sucht das Soziale klassisch bewußtseinstheoretisch, so wie Husserl es in der fünften der Cartesianischen Meditationen vorführte, in einer Gemeinschaft der operational unabhängigen Bewußtseine - und scheitert damit. ${ }^{19}$ Oder aber man sucht es außerhalb der Bewußtseine in einer eigenständige Sphä-

${ }^{19}$ Um die Existenz des Sozialen, hier definiert als 'Intersubjektivität', zu begründen, bleibt Husserl auf der Grundlage der phänomenologischen Epoché nichts anderes übrig, als Intersubjektivität in der Fremderfahrung des einzelnen Subjekts zu fundieren: Ego erfährt Alter Ego als jemanden, der die Welt in gleicher Weise erfährt als er selber. Die Einstimmigkeit der Bewußtseinsoperationen, der Monadengemeinschaft kann dann für die Bewußtseinsphilosophie jedoch immer nur ein intentionales Produkt des einzelnen BewuBtseins sein - und niemals als ein tatsächlich bewußtseinstranszendierendes Phänomen gedacht werden (Husserl 1931: 91-155). re des Sozialen - nur dann ist man erfolgreich. Da die Handlungstheorien den zweiten Weg erkennbar nicht gehen, müssen sie umgekehrt für Luhmann offenbar den ersten Weg, den der klassischen Bewußtseinsphilosophie beschreiten. Bewußtsein und Außenwelt operational unterscheiden und sich damit in die bekannten subjektphilosophischen Probleme verstricken.

Dieser Einwand mag für bestimmte Typen der Handlungstheorie zutreffen. ${ }^{20}$ Luhmann nimmt jedoch für alle 'handlungstheoretischen' Ansätze und damit auch für jene, die von Wittgenstein und vom Strukturalismus ausgehen, eine InnenAußen-Differenz von Bewußtsein und Sozialwelt an, die diese nicht als gültiges Differenzschema anerkennen. Da die Kulturtheorien nicht operationalistisch denken, setzen sie keine operative Differenz zwischen 'inneren' Gedanken und 'äußeren' Kommunikation voraus. Damit stellt sich aber auch nicht die Alternative, das Soziale entweder im einzelnen Bewußtsein oder mit Luhmann auBerhalb des Bewußtseins in den sozialen Systemen zu suchen. Wenn die Kulturtheorien von Bourdieu und Giddens auf jener Dezentrierung des Subjekts und der Sozialisierung des Bewußtseins aufbauen, wie sie von Wittgenstein und Saussure vollzogen wurden, gehen sie damit vielmehr von einer Vorstellung des Sozialen aus, die unabhängig von einer Innen-Außen-Differenz besteht. Das Soziale erscheint hier nicht als eine spezifische Operation, sondern als eine praxisermöglichende Struktur: das Soziale sei in den sinnhaften Regeln fundiert, die die Akteure in Form von Wissensstrukturen in ihren sozialen Praktiken anleiten. Die Sozialwelt sei in Form von Wissensregeln gewissermaßen in den Akteuren 'inkorporiert', und die Akteure weit entfernt davon, als klassische Subjekte begriffen zu werden - vermögen umgekehrt nur auf der Grundlage dieses sozialisierten Wissens zu agieren. Damit werden in den Kulturtheorien die Bewußtseine nicht als gegebene, nur der eigenen Introspektion zugängliche, 'privatsprachliche' Entitäten beschrieben, sondern erscheinen im Gegenteil durch ihre Einbettung in kollektive Wissensstrukturen, die die jeweiligen Lebensformen bilden, eigentümlich 'dezentriert'.

Die Kulturtheorien stehen damit in ihrer Vorstellung des Sozialen als praxisanleitenden Wissens-

${ }^{20}$ Das beste Beispiel für eine tatsächlich an Husserls BewuBtseinsphilosophie anknüpfende Handlungstheorie und die sich damit ergebenden Probleme - ist der frühe Alfred Schütz in seiner Arbeit 'Der sinnhafte Aufbau der sozialen Welt'. 
strukturen auch den klassischen, intentionalistischen Handlungstheorien entgegen, die das Soziale dem 'sozialen Handeln' zurechnen, mithin von der sozialen Orientierung des subjektiven Sinns des Handelnden abhängig machen. ${ }^{21}$ Wenn für die Kulturtheorien hingegen nicht die Art der Handlungsintention für die Sozialität des Handelns verantwortlich ist - was tatsächlich auf jene von Luhmann kritisierte Subjektivierung des Sozialen hinausläuft -, sondern jedem Handeln ein kollektives Regelwissen zugrundeliegt, betrifft dies nicht allein die Fälle 'sozialen', am Handeln anderer orientierten Handelns, sondern auch etwa instrumentell-objektorientierte und motorische Handlungsweisen ${ }^{22}$ Tatsächlich beruht die Konzeption wissensangeleiteter Praktiken damit nicht auf einem individualistischen Denken, welches soziale Eigenschaften auf individuelle Eigenschaften rückführen wollte, sondern auf dem eines sozialen Holismus, das von der impliziten Regelabhängigkeit der Akteure und ihres Handelns ausgeht.

Interpretiert man Luhmanns Ansatz als Version einer Innen-Außen-Differenz, so wird jedoch umgekehrt deutlich, daß ein gängiges Vorurteil gegenüber der Systemtheorie sich ebenso wenig aufrechterhalten läßt: der Vorwurf des sich aus dem 'Holismus' der Systemtheorie ergebenden 'AntiIndividualismus'. Tatsächlich steht Luhmanns Begriff eines sozialen Systems als einer sich selbst reproduzierenden Operation, in diesem Sinne einer emergenten Ebene des Sozialen in strikter Gegnerschaft zu jedem 'Methodologischen Individualismus'. Wenn man jedoch deutlich macht, daß Luhmanns Ansatz auf der Bifurkation von sozialen und psychischen Systemen beruht, so wird die

${ }^{21}$ Diese Position ist bekanntlich von Max Weber in den 'Soziologischen Grundbegriffen' vertreten worden (1922: 11f).

22 Damit ist ein interessanter Punkt berührt, der hier nicht genauer verfolgt werden kann: die Folgen der Luhmann'schen Entscheidung, nicht Handeln, sondern Kommunikation als basale 'Operation' zu wählen. Dies erscheint Luhmann notwendig, da nur Kommunikation als Operation verspricht, schon per definitionem 'sozial' zu sein, während Handeln, insbesondere in den Fällen instrumentell-objektorientierten Handelns (Luhmanns Beispiel hier: Zähneputzen; 1992c: Vorlesung 4) auch nicht sozial sein könne. Für die Kulturtheorien stellt sich diese Frage jedoch nicht in dieser Weise: wenn das Soziale des Handelns nicht in dem subjektiven Handlungssinn, sondern in den kollektiv wirkenden Wissensregeln zu suchen ist, die dieses Handeln anleiten, dann ist instrumentelles Handeln genauso sozial fundiert wie Kommunikationshandeln: auch Zähneputzen ist eine kulturspezifische soziale Praktik. 'individualistische', die nicht nur im übertragenen, sondern im wörtlichen Sinne bewußtseinsphilosophische Kehrseite des Konzepts des sozialen Systems offensichtlich, die sich hinter dem Konzept des psychischen Systems verbirgt. ${ }^{23}$ Exakt wie in der Husserl'schen Phänomenologie will Luhmann das psychische System als inneres Bewußtseinsleben verstanden wissen, das auf der Grundlage seines eigenen, aus keinem Äußeren ableitbaren Sinnhorizontes operiert. Konsequenter als dies in Husserls Cartesianischen Meditationen geschieht, soll das Bewußtsein zudem nicht in eine 'Monadengemeinschaft' der Bewußtseine eingeordnet, sondern tatsächlich als eine autopoietisch sich selbst reproduzierende, im modernen Sinne 'individuelle' Beobachtungsperspektive respektiert werden. Damit vertritt Luhmann gerade keinen alles umfassenden sozialen Holismus - wie er etwa von Parsons formuliert wird, wenn er von einer Internalisierung der sozialen Werte und Normen in die Akteure ausgeht -, sondern parallel zu seiner Theorie sozialer Systeme eine Theorie des autonomen, autopoietischen Bewußtseins. Nimmt man die Bifurkation zwischen psychischen und sozialen Systemen ernst und versteht beide als irreduzible Typen von Beobachtungsoperationen, stellt sich Luhmanns Ansatz damit plakativ gesprochen zur Hälfte als 'holistisch', zur Hälfte als 'individualistisch' heraus: Soziale Systeme lassen sich 'holistisch' als irreduzible soziale Kommunikationen begreifen, psychische Systeme 'individualistisch' als irreduzible individuelle Gedankensequenzen. Es ist letztlich eine Frage der Perspektive, eine Frage der Systemreferenz, ob man von den sozialen Operationen oder den Gedanken ausgeht. Keiner der beiden Operationen erscheint für Luhmann durch die jeweils andere präformiert oder determiniert (höchstens irritieren sie einander) -

${ }^{23}$ In einem übertragenen Sinne kann Luhmann nicht nur sein Konzept des psychischen Systems, sondern sein gesamtes Systemkonzept als 'individualistisch' bezeichnen: die operative Schließung jedes Systems ermögliche dessen Einzigartigkeit und damit Individualität (vgl. 1994: 165). Man muß hier aber sorgfältig zwischen drei verschiedenen Bedeutungen des Terminus 'Individualismus' unterscheiden: 1) Mit dem gängigen Begriff des 'Methodologischen Individualismus' wird eine allgemeine Reduktion von Sozialem auf Individuelles bezeichnet - eine Position der sowohl die System- als auch die Kulturtheorien entgegenstehen. 2) In Luhmanns eigener Begriffsverwendung wird 'Individualismus' zur Charaktierisierung des Systemkonzepts des operativen Konstruktivismus benutzt. 3) Wir wollen hier mit dem Begriff des Individualismus hingegen auf eine Fassung des Konzepts des Bewußtseins verweisen, die in der Tradition der Subjektphilosophie steht. 
dies gilt auch für die psychischen Systeme und für ihre 'Individualität'.

Aus der Sicht einer an Wittgenstein und Saussure geschulten Kulturtheorie werden Fragen damit nicht an den vermeintlich subjektfeindlichen Holismus von Luhmanns Systemtheorie zu richten sein, jedoch umgekehrt an deren 'individualistische' Elemente. Nicht eine 'Marginalisierung' des Individuums ist das Merkmal der Systemtheorie, sondern die Anlehnung des Konzepts des psychischen Systems an die Bewußtseinsphilosophie, damit letztendlich die A-priori-Individualisierung des Subjekts als eines autopoietisch-selbstreferentiellen Bewußtseins. Wenn man philosophiehistorisch argumentieren wollte, könnte man auf die in der neueren Philosophiegeschichte angeführten kritischen Argumente gegen die Bewußtseinsphilosophie, formuliert von Peirce und Saussure bis Heidegger und Wittgenstein und dessen Nachfolger, zurückgreifen und diese Kritik auf Luhmanns Begriff des psychischen Systems anwenden. ${ }^{24}$ Probleme, die sich aus Luhmanns Bifurkation zwischen Psychischem und Sozialem für sozialwissenschaftliche Analysen ergeben können, kann man jedoch auch konkret festzumachen versuchen, wenn man die Systemtheorie vor dem Hintergrund des Analyserahmens der Kulturtheorien betrachtet. Ein einzelnes, aber exemplarisches Problem soll hier herausgegriffen werden: der Wissensbegriff. Die Innen-Außen-Differenz zwischen psychischen und sozialen Systemen bei Luhmann führt offenbar zu einer Engführung des Begriffs des sozialen Wissens auf semantisches Wissen und einer Zurechnung von nicht-semantischem, knowing how-förmigem Wissen auf psychische Systeme, das damit nicht mehr als soziales, kollektives Wissen behandelt werden kann.

Für die Kulturtheorien und die an sie anschließenden Analysen bildet kollektives Wissen als ,shared knowledge" ein Feld von all dem, ,what (people) must know in order to act as they do, making things they make, and interpret their experiences in the distinctive way they do." (Quinn/ Holland 1987: 4) Diese Wissensstrukturen umfassen sowohl semantisches Wissen, das heißt Wissen um

${ }^{24}$ Luhmann selber erwähnt nur an einer Stelle die mögliche Angreifbarkeit eines bewußtseinstheoretischen Konzepts des psychischen Systems, wenn er vielsagend formuliert: „Ob und wie man damit [mit einer Theorie autopoietischer psychischer Systeme] aus bekannten Schwierigkeiten einer Philosophie des selbstreferentiellen Bewußtseins (etwa Fichtescher Prägung) herauskommt, muß einer späteren Prüfung überlassen bleiben.“ (1984: 355). die Bedeutung von Wörtern, Sätzen und ihre Verwendung, als auch nicht-semantisches Wissen. Nicht-semantisches Wissen läßt sich wohl am besten unter Gilbert Ryles Oberbegriff des knowing how (im Gegensatz zum knowing that) zusammenfassen: Knowing how-Wissen umfaßt, Ryle folgend, 'Handlungskriterien', 'Dispositionen' und 'Regeln', die ,the ability ... to do certain sorts of things" (28) begründen (Ryle 1949: 13- 60, 112147) und die damit Ähnliches wie das bezeichnen, was Schütz unter dem Oberbegriff des 'Routinewissens' als Fertigkeiten, Gebrauchs- und Rezeptwissen umschreibt (Schütz/Luckmann: 1975). Entscheidend für die Kulturtheorien ist, daß auch dieses knowing how, das in semantischem Wissen nicht aufgeht - die Fähigkeit Schach zu spielen, zu schreiben, sich gegenseitig mit Höflichkeit zu begegnen etc. -, ein kollektives Wissen darstellt, welches soziale Reproduktion ermöglicht. ${ }^{25}$

Wo sind diese Phänomene in Luhmanns Theoriegebäude zu finden? Es hat den Anschein, daß die Differenz zwischen sozialen und psychischen Systemen Luhmann zu einer Fassung des Wissensbegriffs zwingt, die das kollektive knowing how-Wissen zu einer Art ausgeschlossenem Dritten macht, das sich weder der einen noch der anderen Seite, weder den Sinnmustern des sozialen noch denen des psychischen Systems zurechnen läßt. Wie dargestellt, ist auch für Luhmann der Begriff des 'Wissens' zentral, erhält aber eine andere Stoßrichtung als der Wissensbegriff der Kulturtheorien. Soziales Wissen, das heißt nicht-subjektives Wissen ist für Luhmann eindeutig den sozialen Systemen zuzurechnen, nicht den psychischen Systemen. Da soziale Systeme aber in Form von Kommunikationen operieren, muß soziales Wissen kommunikationsstrukturierendes Wissen sein und wird von Luhmann daher konsequent als Semantik identifiziert (1990: 107). Die Identifikation von sozialem Wissen bzw. der Struktur von Kommunikation mit Semantik ist nicht zufällig, sondern ergibt sich zwingend aus dem Theoriedesign: Wenn Kommunikation in aller Regel sprachliche Kommunika-

${ }^{25}$ Die hier verwendete Unterscheidung zwischen semantischem und nicht-semantischem Wissen scheint auf den ersten Blick mit Polanyis Differenz zwischen implizitem und explizitem Wissen verwandt zu sein. Letztlich scheint eine Gleichsetzung dieser beiden Unterscheidungen jedoch nicht sinnvoll: Während nicht-semantisches knowing how-Wissen tatsächlich im wesentlichen 'implizit' bleibt, gilt im Umkehrschluß nicht die Explizitheit des semantischen Wissen. Im Gegenteil ist auch dieses - so wie Searle es etwa in seiner Theorie der 'Präsuppositionen' darstellt - zum großen Teil 'implizit'. 
tion ist, dann muß auch die Sinnstruktur, die diese sprachliche Kommunikation anleitet, eine sprachliche, damit eine semantische Sinnstruktur sein.

Der Ort von nicht-semantischem Wissen scheint dann nur konsequent: Es bleiben die einzelnen psychischen Systeme. Luhmann bringt die Unterscheidung zwischen dem (semantischen) Wissen von Sozialsystemen und dem 'Wissen' psychischer Systeme mit Polanyis Differenz zwischen explizitem und implizitem Wissen in Zusammenhang und stellt fest: „Ein Beobachter kann das System ... mit Hilfe der Unterscheidung explizit/ implizit beobachten und beschreiben. Er kann in das, was als Wissen geschieht, zusätzlich die strukturellen Kopplungen hineinsehen" (1990: 42). Aus der Perspektive der sozialen Systeme heißt dies dann: gleichzeitig zu dem in der Kommunikation verarbeiteten semantischen Wissen existieren in der Umwelt der - dann 'angekoppelten' - psychischen Systemen jeweils andere Wissensbestände. (1990: 41-44) Es gibt also auch für Luhmann Wissen auBerhalb der sozialen Systeme und ihrer Semantik (wobei hier der Wissensbegriff vermieden wird): Wenn soziales Wissen semantisches Wissen ist, dann scheint die Schlußfolgerung berechtigt, daß bei Luhmann nicht-semantisches Wissen, das Ryles knowing-how ähnelt, den einzelnen psychischen Systemen zuzurechnen ist. Beispielsweise „kann das Bewußtsein im Fortschreiten von Gedanken zu Gedanken sich auf bestimmtes Können verlassen..., ohne darüber bewußt entscheiden zu müssen." (1990: 43) ${ }^{26}$ Abgesehen davon, da $\beta$ es Schwierigkeiten machen würde, diese Vorstellung mit dem Konzept des knowing how vollends zu identifizieren - dieser Begriff bezieht sich bei Ryle schließlich gerade nicht auf Bewußtseinsprozesse, sondern auf aktivierte Handlungskriterien und Dispositionen - muß man nun aber an dieser Stelle die Einsicht wiederaufgreifen, daß psychische Systeme für Luhmann jeweils individuelle Systeme bilden, die nur dem eigenen Ich zugänglich sind: Jedes dieser Bewußtseine stellt eine eigenständige Operationssequenz dar, jedes verfügt über seine eigenen Beobachtungsschemata, deren Ausbildung durch Umweltereignisse höchstens 'irritiert' werden kann. Das 'nicht mitkommunizierte' 'Wissen' der psychischen Systeme muß für Luhmann also zwangsläufig individuelles, bewußtseinsspezifisches Wissen sein. Alle Versuche, im Rahmen des

${ }^{26}$ Allerdings scheint Luhmanns Darstellung an dieser Stelle es nahezulegen, daß dieses knowing how wiederum als eine strukturelle Kopplung von psychischen und organischen Systemen zu begreifen ist. autopoietisch-selbstreferentiellen Verständnisses des Bewußtseins die Gedankenfigur eines gemeinsamen, sozialen Wissens der psychischen Systeme einzubauen, müssen nach Luhmanns Urteil genauso scheitern, wie Husserls Versuch, in seine Bewußtseinsphilosophie doch noch gewissermaßen nachträglich die Figur einer Intersubjektivität und „Monadengemeinschaft" einzufügen. Damit ergibt sich jedoch folgendes Bild: Soziales Wissen ist semantisches Wissen und der Gegenstand soziologischer Wissensanalyse. Nicht-semantisches 'Wissen' nach Art des kulturtheoretischen knowing how kann dann umgekehrt nur in den psychischen Systemen einen Platz finden - muß dann jedoch als individuelles Wissen der 'sechs Milliarden verschiedenen' Bewußtseine gedacht werden und wäre damit für die Soziologie wohl verloren.

Nun kann kein Zweifel darüber bestehen, daß ein Teil des sozial relevanten Wissens einer Gesellschaft in der Form von Semantik auftritt, da $B$ die Analyse der semantischen Bestände für eine Analyse der Kultur und des Wissens einer Gesellschaft von beträchtlicher Relevanz ist. Jedoch haben kulturtheoretisch motivierte Analysen regelmäßig darauf hingewiesen, $\mathrm{da} B$ man dieses Wissen um die Verwendung von Wörtern und Sätzen als die 'Spitze des Eisberges' betrachten muß, daß die Reproduktion der sozialen Welt ohne die Existenz und permanente Anwendung knowing how-förmiger und dabei ebensosehr kollektiver - Wissensbestände kaum begreifbar wäre. Wenn Bourdieu die verschiedenen Ausformungen des 'praktischen Sinns' untersucht, die scheinbar zwanglos die jeweiligen Vorlieben und Abneigungen im Konsum unterschiedlicher sozialer Klassen hervorbringen, wenn Goffman die Interaktionsregeln herausarbeitet, die wirken, damit jedes Ich sein Gesicht vor den anderen wahren kann, wenn Schütz und Luckmann die universalen Schemata zur Konstitution des sozialen Raums und der sozialen Zeit beschreiben, wenn schließlich Garfinkel die Fertigkeiten rekonstruiert, die nötig sind, damit eine Person 'geschlechtsadäquates' Verhalten hervorzubringen vermag, so liegen in allen Fällen Analysen von sozialem knowing how-Wissen vor, von Sinnmustern, die kollektiv wirksam sind, ohne mit semantischem Wissen identisch zu sein (Bourdieu 1979; Goffman 1971; Schütz/ Luckmann 1975: 6387; Garfinkel 1967: 116-185). Kollektives knowing how-Wissen liefert dabei mehr als bloß ein mehr oder minder interessantes Untersuchungsobjekt: aus der Sicht der Kulturtheorien wäre soziale Reproduktion, wäre die Repetitivität der menschlichen Praktiken, die letztlich der sozialen Welt 
ihre relative Geordnetheit verleiht, ohne sie nicht denkbar.

Die Innen-Außen-Differenz zwischen sozialen und psychischen Systemen zwingt Luhmann jedoch zu einer eindeutigen Zurechnung von Wissen auf Kommunikation und damit zu einer Identifizierung von Wissen und Semantik. Zwar wird bei ihm, wie dargestellt, eine Hilfskonstruktion genannt: die strukturelle Kopplung zwischen Kommunikation und Bewußtsein, ,...die strukturelle Kopplung dieser sequentiellen [kommunikativen] Ereignisse mit Bewußtseinszuständen, die nicht mitkommuniziert werden." (1990: 43) Der Begriff der strukturellen Kopplung soll mithin das wieder zusammenbinden, was vorher als operational getrennt voraussetzt worden war: die sozialen Systeme und die psychischen Systeme, damit das semantische Wissen und das aus Sicht der Sozialsysteme 'implizite Wissen' der einzelnen psychischen Systeme. Der Begriff der strukturellen Kopplung wirft jedoch in diesem Zusammenhang offensichtlich zwei Probleme auf:

Wenn man der Selbstlimitation des autopoietischen Theoriedesigns folgt, kann der Begriff 'strukturelle Kopplung' keine Abhängigkeits- oder Generierungsbeziehung, sondern nichts anderes als ein Irritationsverhältnis zwischen Kommunikation und Bewußtseinen behaupten. Für ein solches Irritationsverhältnis gilt jedoch, wie bereits festgestellt, daß ein System sich nur nach Maßgabe der eigenen Sinnschemata von einem fremden System irritieren lassen kann. Das heißt aber, daß semantisch angeleitete Kommunikationen sich nur dann vom knowing how-Wissen einzelner psychischer Systeme irritieren lassen können, wenn das, was dort implizit vorhanden ist, in irgendeiner Weise in die Kommunikation des sozialen Systems übersetzt wird - dann aber kein knowing how mehr darstellt. Eine direkte Ermöglichung bzw. Einschränkung von sozialen Praktiken auch durch nicht-semantisches Wissen, wie in den Kulturtheorien gedacht, erscheint damit nicht möglich. Vor allem aber ändert auch der Begriff der strukturellen Kopplung nichts an der Vorannahme, daß dieses nicht-semantische im Unterschied zum semantischen Wissen einzelnen psychischen und damit individuellen Systemen zugerechnet bleiben muß, selber per definitionem keine kollektiv-soziale Qualität haben und in diesem Sinne wohl kein direktes Untersuchungsobjekt der Sozialwissenschaften bilden kann. Die klassisch-subjektphilosophische Fassung des Bewußtseins und damit auch jenes 'Wissens', das dort zugerechnet wird, kann durch den Begriff der strukturellen Kopp- lung nicht modifiziert, sondern nur bestätigt werden.

Es scheint, daß die Bifurkation von Bewußtsein und Kommunikation Luhmann zu einer 'Intellektualisierung' des Begriffs sozialen Wissens führt. Die Kulturtheorien folgen in ihrem Verständnis von Wissen jener Umdeklinierung des Wissensbegriffs vom knowing that auf ein knowing how, wie sie Ryle in seiner Kritik der Subjektphilosophie im Kielwasser Wittgensteins angemahnt hatte. Für Luhmann bleiben die Strukturen des sozialen Wissens, wenn sie schon keine Gedankeninhalte mehr sind, hingegen allein semantische Bestände der Kommunikationssysteme. Die Ausgangsdifferenz von Innen und Außen, zwischen Sozialwelt und Bewußtsein scheint zu dieser Festlegung zu zwingen.

Wenn man, Luhmann folgend, „Kultur“ in einem ganz allgemeinen Sinn als ,eine Perspektive für die Beobachtung von Beobachtern" (1995a: 54) auffaßt, dann liefern alle Typen sinnverstehender Sozialwissenschaft im weitesten Sinne 'Kulturanalysen': Sowohl die konstruktivistische Systemtheorie als auch jene Theorien wissensangeleiteter sozialer Praktiken, die wir als Kulturtheorien umschrieben haben, bieten Rekonstruktionen sozialer Sinnmuster und Sinnprozesse und damit 'Beobachtungen von Beobachtungen'. Die Frage ist dann, von welchen - notwendigerweise kontingenten - begrifflichen Unterscheidungen sich eine solche sinnorientierte Sozialtheorie leiten lassen sollte. Das Muster einer Innen-Außen-Unterscheidung zwischen Sozialwelt und Bewußtsein liefert eine, im modernen Denken durchaus traditionsreiche Option. Die Sabotierung dieser Innen-AußenDifferenz stellt eine andere, zumindest im Denken des 20. Jahrhunderts nicht minder traditionsreiche dar. Allein die gegenseitige Beobachtung beider Theorievokabulare vermag wohl die notwendigen immanenten Limitationen der beiden Unterscheidungssysteme aufzudecken.

\section{Literatur}

Bauman, Zygmunt, 1973: Culture as praxis, London/ New York: Routledge

Bohman, James/ David Hiley/ Richard Shusterman, 1991: The Interpretive Turn, Ithaca: Cornell University

Bourdieu, Pierre, 1970: Zur Soziologie der symbolischen Formen, Frankfurt/ Main 1991: Suhrkamp

Bourdieu, Pierre, 1972: Entwurf einer Theorie der Praxis (auf der ethnologischen Grundlage der kabylischen Gesellschaft), Frankfurt/ Main 1979 (frz.: Esquisse d'une théorie de la pratique, précédé de trois études d'éthnologie kabyle): Suhrkamp 
Bourdieu, Pierre, 1979: Die feinen Unterschiede. Kritik der gesellschaftlichen Urteilskraft, Frankfurt/ Main 1989 (frz.: La distinction. Critique sociale du jugement): Suhrkamp

Bourdieu, Pierre, 1980: Sozialer Sinn. Kritik der theoretischen Vernunft. Frankfurt/ Main 1987 (frz.: Le sens pratique): Suhrkamp

Bourdieu, Pierre, 1994: Raisons pratiques. Sur la théorie de l'action. Paris: Seuil

Bourdieu, Pierre/ Loic J.D. Wacquant, 1992: Réponses. Pour une anthropologie réflexive, Paris: Seuil

Chaney, David. 1994: The Cultural Turn. Scene-setting essays on contemporary cultural history, London/ New York: Routledge

Cohen, Ira J., 1991: Theories of Action and Praxis. S. 111142 in: Bryan S. Turner $(\mathrm{Hg}$.): The Blackwell Companion to Social Theory, Oxford: Blackwell

Descartes. René, 1641: Meditationen über die Grundlagen der Philosophie, Hamburg 1993 (lat.: Meditationes de prima philosophia): Meiner

Durkheim. Émile, 1895: Die Regeln der soziologischen Methode, Frankfurt/ Main 1984 (frz.: Les règles de la méthode sociologique): Suhrkamp

Ellrich, Lutz, 1992: Die Konstitution des Sozialen. Phänomenologische Motive in N. Luhmanns Systemtheorie. Zeitschrift für philosophische Forschung: 24-43

Englisch, Felicitas, 1991: Strukturprobleme der Systemtheorie - Philosophische Reflexionen zu Niklas Luhmann. S. 196-235 in: Stefan Müller-Doohm (Hg.): Jenseits der Utopie. Theoriekritik der Gegenwart, Frankfurt/ Main: Suhrkamp

Garfinkel, Harold, 1967: Studies in Ethnomethodology, Cambridge 1984: Polity

Giddens, Anthony, 1976: New Rules of Sociological Method. A positive critique of interpretative sociologies, Cambridge 1993, 2. Aufl.: Polity

Giddens, Anthony, 1979: Central Problems in Social Theory. Action, structure and contradiction in social analysis, London: Macmillan

Giddens, Anthony, 1984: The Constitution of Society. Outline of the theory of structuration, Cambridge: Polity

Giddens, Anthony, 1987: Social Theory and Modern Sociology, Cambridge: Polity

Goffman, Erving, 1971: Das Individuum im öfentlichen Austausch. Mikrostudien zur öffentlichen Ordnung, Frankfurt/ Main 1982 (engl.: Relations in Public. Microstudies of the public order): Suhrkamp

Grathoff, Richard, 1989: Milieu und Lebenswelt. Einführung in die phänomenologische Soziologie und die sozialphänomenologische Forschung, Frankfurt/ Main: Suhrkamp

Habermas, Jürgen, 1971: Vorlesungen zu einer sprachtheoretischen Grundlegung der Soziologie. S.11-126 in: ders.: Vorstudien und Ergänzungen zur Theorie des kommunikativen Handelns, Frankfurt/ Main 1984: Suhrkamp

Habermas, Jürgen, 1985: Der philosophische Diskurs der Moderne. Zwölf Vorlesungen, Frankfurt/ Main 1991, 3. Aufl.: Suhrkamp
Harland. Richard. 1987: Superstructuralism, London/ New York: Routledge

Husserl, Edmund, 1931: Cartesianische Meditationen. Eine Einleitung in die Phänomenologie, in: Gesammelte Schriften 8, Hamburg 1992: Meiner

Luhmann, Niklas, 1984: Soziale Systeme. Grundriß einer allgemeinen Theorie. Frankfurt/ Main 1991, 4. Aufl.: Suhrkamp

Luhmann. Niklas, 1986a: Die Lebenswelt - nach Rücksprache mit Phänomenologen. Archiv für Rechtsund Sozialphilosophie: 176-194

Luhmann. Niklas, 1986b: Intersubjektivität oder Kommunikation: Unterschiedliche Ausgangspunkte soziologischer Theoriebildung. S. 169-188 in: Luhmann 1995b

Luhmann, Niklas, 1987a: Archimedes und wir. Interviews, Berlin: Merve

Luhmann, Niklas, 1987b: Die Autopoiesis des Bewußtseins. S. 55-112 in: Luhmann 1995b

Luhmann, Niklas, 1987c: Die gesellschaftliche Differenzierung und das Individuum. S. 125-141 in: Luhmann $1995 b$

Luhmann, Niklas, 1987d: Was ist Kommunikation? S. 113-124 in: Luhmann 1995b

Luhmann, Niklas, 1988: Wie ist Bewußtsein an Kommunikation beteiligt? S. 37-54 in: Luhmann 1995b

Luhmann, Niklas, 1989: Gesellschaftsstruktur und Semantik. Studien zur Wissenssoziologie der modernen Gesellschaft, Band 3, Frankfurt/ Main 1993: Suhrkamp

Luhmann, Niklas, 1990: Die Wissenschaft der Gesellschaft, Frankfurt/ Main: Suhrkamp

Luhmann, Niklas, 1991: Die Form 'Person'. S. 142-154 in: Luhmann 1995b

Luhmann, Niklas. 1992a: Beobachtungen der Moderne, Opladen: Westdeutscher Verlag

Luhmann, Niklas, 1992b: Die operative Geschlossenheit psychischer und sozialer Systeme. S.25-36 in: Luhmann 1995b

Luhmann, Niklas, 1992c: Einführung in die Systemtheorie. Vorlesung vom Wintersemester 1991/92, Universität Bielefeld, in 14 Hörcassetten, Heidelberg: Carl Auer

Luhmann, Niklas, 1992d: Wer kennt Will Martens? Eine Anmerkung zum Problem der Emergenz sozialer Systeme. Kölner Zeitschrift für Soziologie und Sozialpsychologie: 139-142

Luhmann, Niklas, 1994: Die Tücke des Subjekts und die Frage nach den Menschen. S. 155-168 in: Luhmann $1995 b$

Luhmann, Niklas, 1995a: Gesellschaftsstruktur und Semantik. Studien zur Wissenssoziologie der modernen Gesellschaft, Band 4, Frankfurt/Main: Suhrkamp

Luhmann, Niklas, 1995b: Soziologische Aufklärung 6. Die Soziologie und der Mensch, Opladen: Westdeutscher Verlag

Luhmann, Niklas, 1996: Die neuzeitlichen Wissenschaften und die Phänomenologie, Wien: Picus

Lukes, Steven 1973: Emile Durkheim: His Life and Work. A historical and critical study, London 1988: Penguin

Martens, Wil, 1991: Die Autopoiesis sozialer Systeme. Kölner Zeitschrift für Soziologie und Sozialpsychologie: $625-646$ 
Plessner, Helmuth, 1928: Die Stufen des Organischen und der Mensch. Einleitung in die Philosophische Anthropologie, Berlin/ New York 1975: de Gruyter

Polanyi, Michael, 1966: Implizites Wissen, Frankfurt/ Main 1985 (engl.: The Tacit Dimension): Suhrkamp

Quinn, Naomi/ Dorothy Holland, 1987: Culture and cognition. S. 3-40 in: dies. ( $\mathrm{Hg}$.): Cultural Models in Language and Thought, Cambridge: Cambridge University Press

Rabinow, Paul/ William M. Sullivan (Hg.), 1979: Interpretive Social Science, Berkeley: University of California

Ritzer, George, 1975: Sociology: A Multiple Paradigm Science, Boston: Allyn and Bacon

Roth, Gerhard, 1987: Autopoiesis und Kognition. Die Theorie H.R. Maturanas und die Notwendigkeit ihrer Weiterentwicklung. S. 256-286 in: Schmidt 1987b

Rubinstein, David, 1981: Marx and Wittgenstein. Social praxis and social explanation, London/ New York: Routledge

Ryle, Gilbert, 1949: The Concept of Mind, London 1990: Penguin

Saussure, Ferdinand de, 1916: Cours de linguistique générale, Paris 1985: Payot

Schmidt, Siegfried J., 1987a: Der Radikale Konstruktivismus: Ein neues Paradigma im interdisziplinären Diskurs. S. 11-88 in: Schmidt 1987b
Schmidt, Siegfried J. (Hg.), 1987b: Der Diskurs des Radikalen Konstruktivismus, Frankfurt/ Main: Suhrkamp

Schütz, Alfred, 1957: Das Problem der transzendentalen Intersubjektivität bei Husserl. S. 86-118 in: Gesammelte Ausätze Band 3, Den Haag 1971: Nijhoff

Schütz, Alfred/ Thomas Luckmann, 1975: Strukturen der Lebenswelt, Band 1, Frankfurt/Main 1991, 4. Aufl.: Suhrkamp

Turner, Stephen P., 1994: The Social Theory of Practices. Tradition, tacit knowledge and presuppositions, Cambridge: Polity

Wagner, Gerhard/ Heinz Zipprian, 1992: Identität oder Differenz? Bemerkungen zu einer Aporie in Niklas Luhmanns Theorie selbstreferentieller Systeme. Zeitschrift für Soziologie: 394-405

Weber, Max, 1922: Wirtschaft und Gesellschaft. Grundriß der verstehenden Soziologie, Tübingen 1980, 5, revidierte Aufl.: Mohr

Wittgenstein, Ludwig, 1953: Philosophische Untersuchungen. S. 225-580 in: Werkausgabe Band 1, Frankfurt/ Main 1984: Suhrkamp

Wittgenstein, Ludwig, 1969: Über Gewißheit. S. 113-257 in: Werkausgabe Band 8, Frankfurt/Main 1984: Suhrkamp 